\title{
Aerodynamic versus Ballistic Flight
}

\author{
Jack Denur \\ Electric \& Gas Technology, Inc., Rowlett, TX, USA \\ Email: jackdenur@my.unt.edu
}

How to cite this paper: Denur, J. (2019) Aerodynamic versus Ballistic Flight. Open Journal of Fluid Dynamics, 9, 346-400. https://doi.org/10.4236/ojfd.2019.94023

Received: January 17, 2017

Accepted: December 15, 2019

Published: December 18, 2019

Copyright $\odot 2019$ by author(s) and Scientific Research Publishing Inc. This work is licensed under the Creative Commons Attribution International License (CC BY 4.0).

http://creativecommons.org/licenses/by/4.0/

\begin{abstract}
We consider, compare, and contrast various aspects of aerodynamic and ballistic flight. We compare the energy efficiency of aerodynamic level flight at a given altitude versus that of ballistic flight beginning and ending at this same altitude. We show that for flights short compared to Earth's radius, aerodynamic level flight with lift-to-drag ratio $L / D>2$ is more energy-efficient than ballistic flight, neglecting air resistance or drag in the latter. Smaller $L / D$ suffices if air resistance in ballistic flight is not neglected. For a single circumnavigation of Earth, we show that aerodynamic flight with $L / D>4 \pi$ is more energy-efficient than minimum-altitude circular-orbit ballistic spaceflight. We introduce the concept of gravitational scale height, which may in an auxiliary way be helpful in understanding this result. For flights traversing $N$ circumnavigations of Earth, if $N \gg 1$ then even minimum-altitude circular-orbit ballistic spaceflight is much more energy-efficient than aerodynamic flight because even at minimum circular-orbit spaceflight altitude air resistance is very small. For higher-altitude spaceflight air resistance is even smaller and the energy-efficiency advantage of spaceflight over aerodynamic flight traversing the same distance is therefore even more pronounced. We distinguish between the energy efficiency of flight per se and the energy efficiency of the engine that powers flight. Next we consider the effects of air density on aerodynamic level flight and provide a simplified view of drag and lift. We estimate the low-density/high-altitude limits of aerodynamic level flight (and for comparison also of balloons) in Earth's and Mars' atmospheres. Employing Mars airplanes and underwater airplanes on Earth (and hypothetically also on Mars) as examples, we consider aerodynamic level flight in rarefied and dense aerodynamic media, respectively. We also briefly discuss hydrofoils. We appraise the optimum range of air densities for aerodynamic level flight. We then consider flights of hand-thrown projectiles that are unpowered except for the initial throw. We describe how aerodynamically efficient ones (i.e., with large $L / D$ ) such as Frisbees, Aerobies, and boomerangs not only can traverse record horizontal distances, but (along with discuses) also can-since lift exceeds weight at achievable throwing speeds-maintain
\end{abstract}


altitude farther if thrown horizontally against the wind than with it. Then we compare the energy efficiency of surface transportation versus that of both aerodynamic and ballistic flight.

\section{Keywords}

Aerodynamic Flight, Ballistic Flight, Lift, Drag, Lift-to-Drag Ratio, Air Density, Energy Efficiency

\section{Introduction}

We consider, compare, and contrast various aspects of aerodynamic and ballistic flight. In Section 2 we compare the energy efficiency of aerodynamic level flight at a given altitude versus that of ballistic flight beginning and ending at this same altitude. We show that for flights short compared to Earth's radius, aerodynamic level flight with lift-to-drag ratio $L / D>2$ is more energy-efficient than ballistic flight, neglecting air resistance or drag in the latter. If air resistance in ballistic flight is not neglected, then smaller $L / D$ suffices for short aerodynamic level flight to be more energy-efficient than short ballistic flight. For a single circumnavigation of Earth (the longest possible flight whose purpose is to reach a destination on Earth, with the destination being the starting point after a round-the-world trip), we show that aerodynamic flight with $L / D>4 \pi$ is more energy-efficient than minimum-altitude single-circular-orbit ballistic spaceflight, neglecting the very small air resistance in the latter. We introduce the concept of gravitational scale height, which may in an auxiliary way be helpful in understanding this result. If the very small air resistance at minimum circular-orbit spaceflight altitude is not neglected, then $L / D$ very slightly smaller than $4 \pi$ suffices for single-circumnavigation aerodynamic flight to be more energy-efficient than minimum-altitude single-circular-orbit ballistic spaceflight. For flights traversing $N$ circumnavigations of Earth, owing to air resistance or drag being very small even at minimum circular-orbit ballistic spaceflight altitude, $L / D$ must exceed $4 \pi N$ for aerodynamic flight to be more energy-efficient than minimum-altitude circular-orbit ballistic spaceflight. But $L / D \approx 100$ may represent the practicable limit that can be achieved even with the most advanced aerodynamic technology. Hence if $N \gg 1$ even minimum-altitude circular-orbit ballistic spaceflight is much more energy-efficient than aerodynamic flight. For higher-altitude spaceflight drag is even smaller and the energy-efficiency advantage of spaceflight over aerodynamic flight traversing the same distance is therefore even more pronounced. In Section 2.4 we distinguish between the energy efficiency of flight per se and the energy efficiency of the engine that powers flight. In Section 3 we consider the effects of air density on aerodynamic level flight and provide a simplified view of drag and lift. We estimate the low-density/high-altitude limits of aerodynamic level flight (and for comparison also of balloons) in Earth's and Mars' atmospheres. Employing Mars airplanes and underwater airplanes on 
Earth (and hypothetically also on Mars) as examples, we consider aerodynamic level flight in rarefied and dense aerodynamic media, respectively. We also briefly discuss hydrofoils. We appraise the optimum range of air densities for aerodynamic level flight. In Section 4 we consider flights of hand-thrown projectiles that are unpowered except for the initial throw. We describe how aerodynamically efficient ones (i.e., with large $L / D$ ) such as Frisbees, Aerobies, and boomerangs not only can traverse record horizontal distances, but (along with discuses) also can-since lift exceeds weight at achievable throwing speeds-maintain altitude farther if thrown horizontally against the wind than with it. In Section 5 we compare the energy efficiency of surface transportation versus that of both aerodynamic and ballistic flight. Brief concluding remarks are given in Section 6. Footnotes provide supporting references and some auxiliary information. Supplementary Notes, providing more comprehensive auxiliary information concerning topics discussed in the main text and/or in the cited references including some additional supporting references, are given in the Appendix.

In this paper we define "aircraft" as any type of aerodynamic flying machine-for example man-made airplane or sailplane, bird, or flying insect in forward or hovering flight, including hand-thrown aircraft (e.g., discus, Frisbee, Aerobie, boomerang, etc.). Underwater airplanes, which we will briefly consider in Section 3.3.2, and hydrofoils, which we will briefly consider in Section 3.4, should be classified as aircraft because their lift obtains aerodynamically rather than via buoyancy, even though the density of their aerodynamic medium (water) is $\approx 800$ times that of air at sea level. By lifting the hull out of water into air, drag on the hull of a hydrofoil at any given speed is reduced $\approx 800$ times; only the wings need suffer water resistance as opposed to air resistance. Except for a few very brief parenthetical remarks concerning hovering flight, in this paper we consider only aircraft that obtain their lift by virtue of their translational forward motion, i.e., translational-lift aircraft-for example man-made airplanes and sailplanes, birds and insects that obtain their lift by virtue of their translational forward motion, underwater airplanes, hydrofoils, and hand-thrown translational-lift aircraft (e.g., discuses, Frisbees, Aerobies, boomerangs, etc.). Except for a brief consideration of underwater airplanes in Section 3.3.2, and a brief consideration in Section 3.4 and occasional other even briefer remarks concerning hydrofoils, from among translational-lift aircraft we consider only aerial translational-lift aircraft, which obtain their lift by virtue of their translational forward motion through air-for example man-made airplanes and sailplanes, birds and insects that obtain their lift by virtue of their translational forward motion, and hand-thrown translational-lift aircraft (e.g., discuses, Frisbees, Aerobies, boomerangs, etc.). ${ }^{1}$

\footnotetext{
${ }^{1}$ Most typically in translational motion an aircraft moves through air. But of course air can be moved past an aircraft, as in a wind tunnel. Also air moved past an aircraft can impart electroaerodynamic propulsion: see Haofeng, X., Yiou, H., Strobel, K.L., Gilmore, C.K., Kelley, S.P., Hennick, C.C., Sebastion, T., Woolston, M.R., Perreault, D.J., and Barrett, S.R.H. (2018) Flight of an aeroplane with solid-state propulsion. Nature, 563, 532-535.
} 
In this paper our main goal is to elucidate more conceptually than mathematically some fundamental ideas concerning energy efficiency and a number of other aspects of aerodynamic versus ballistic flight, and to provide comparison with surface transportation. Some elucidations of this type have, of course, been provided elsewhere. But to the best knowledge of the author, this paper provides a range of such elucidations, and some new ones, not found elsewhere, at least not in a single work. We do not attempt the mathematically complex and detailed fully-quantitative analyses based on rigorous application of fluid dynamics, e.g., computational fluid dynamics, as is required in the actual design of aircraft, or the analyses required in the actual design of spacecraft or surface vehicles. Thus our analyses are qualitative to semiquantitative.

\section{Energy Efficiency of Aerodynamic Versus Ballistic Flight}

\subsection{Short Flights: Aerodynamic Flight Wins}

We compare the energy efficiency of aerodynamic level flight at a given altitude versus that of ballistic flight beginning and ending at this same altitude, at first neglecting air resistance or drag in the case of ballistic flight. We define a short flight as one traversing horizontal distance $X$ much smaller than Earth's radius $R \doteq 6370 \mathrm{~km}$. [The dot-equal sign $(\doteq)$ means "very nearly equal to".] Hence for a short flight Earth's curvature can be neglected. Let $h_{A}$ be the altitude above mean sea level of aerodynamic level flight, and also the beginning or initial altitude $h_{B, \text { initial }}=h_{A}$ and ending or final altitude $h_{B, \text { final }}=h_{A}$ of ballistic flight. (The subscript $A$ denotes aerodynamic flight and the subscript $B$ denotes ballistic flight.) Hence this altitude is at radial distance $r_{A}=R+h_{A}$ from Earth's center. For simplicity we let $h_{A} \ll R$ as is the case for all aerodynamic flight and $h_{B} \ll R$ as is the case for low-altitude short ballistic flight (and even for minimum-altitude circular-orbit ballistic spaceflight).

By elementary Newtonian mechanics [1], a short ballistic flight of a projectile of mass $m$ and weight $m g$, beginning and ending at altitude $h_{B, \text { initial }}=h_{B, \text { final }}=h_{A}$ and traversing horizontal distance $X$ much smaller than Earth's radius $R$, costs energy [1]

$$
E_{B, \text { short }}=\frac{1}{2} m g X .
$$

The local acceleration due to gravity is $g$ and drag is neglected.

An aerodynamic level flight of an aircraft of mass $m$ and weight $m g$ subject to aerodynamic lift $L$ and aerodynamic drag $D$ traversing any horizontal distance $X$, short or long, costs energy

$$
E_{A}=D X=\frac{D}{L} L X=\frac{D}{L} m g X=\frac{m g X}{L / D} .
$$

Of course, for any flight, short or long, "level" and "horizontal" mean that $h_{A}$ and therefore $r_{A}=R+h_{A}$ is constant, a long flight following the curvature of Earth. 
The first step of Equation (2) is justified because to maintain aerodynamic level flight through horizontal distance $X$ air resistance (most generally, fluid resistance) or drag $D$ must be overcome through distance $X$. The third step of Equation (2) is justified because to maintain aerodynamic level flight lift $L$ must equal the weight $m g$ of an aircraft [2]-[15]. ${ }^{2}$ The lift-to-drag ratio $L / D$ of a translational-lift aircraft is the ratio (horizontal distance traversed) $\div$ (altitude lost) in gliding flight without engine power relative to the air (also relative to the ground if the wind is calm) [2]-[15]. ${ }^{2}$ For any translational-lift aircraft $L / D$ is maximized if the aircraft is flown at its most energy-efficient angle of attack. In this paper unless otherwise noted we always assume $L / D$ to be thus maximized. [For auxiliary information, which may also be helpful in some cases wherein Refs. [2]-[15] ${ }^{2}$ are cited later in this paper, see Supplementary Notes 1-6. Also, we discuss these points more thoroughly in Section 3. Wherever helpful, refer to Decker, J.S. (2014) See How It Flies at https://www.av8n.com/how/.]

Comparing Equations (1) and (2), if $L / D>2$, then $E_{A}<E_{B, \text { short }}$. Hence short aerodynamic level flight with $L / D>2$ is more energy-efficient than short ballistic flight beginning and ending at the same altitude, neglecting air resistance in the latter. This requirement $L / D>2$ is met by practically all aircraft (including all birds and flying insects), even by aerodynamically inefficient ones [2]-[15]. Thus short aerodynamic level flight is practically always more energy-efficient-in most cases considerably more energy-efficient-than short ballistic flight, even neglecting air resistance in the latter. But air resistance is not always negligible for short ballistic flight, especially in the lower atmosphere. If it is not neglected, then the requirement is even milder, namely $L / D>2 n$ with $n<1$. Hence not neglecting air resistance in short ballistic flight, short aerodynamic level flight is all the more energy-efficient than short ballistic flight.

By Equations (1) and (2), if air resistance in short ballistic flight can be neglected, then both $E_{B, \text { short }}$ and $E_{A}$ are directly proportional to $m g$. Hence reducing $m g$ reduces the energy cost of both short ballistic flight and short aerodynamic level flight traversing given horizontal distance $X$ equally and in direct proportion to the reduction in $\mathrm{mg}$, but does not alter the ratio of energy costs between these two modes of flight. If air resistance in short ballistic flight cannot be neglected, then reducing $m g$ reduces the energy cost of short ballistic flight less than in direct proportion to the reduction in $\mathrm{mg}$.

Most typically, $m g$ is reduced by reducing $m$. But we can also consider reduction of $g$. Two examples: (i) Aerodynamic level flight on Mars is at lower $g$. (Of course, for Mars $R \doteq 3390 \mathrm{~km}$.) (ii) An aircraft of mass $m$ a fraction $f$ $(0<f<1)$ of whose weight $m g$ is offset by buoyancy can be construed as either being of effective mass $m(1-f)$ in a gravitational field $g$ or as being of mass $m$ in a gravitational field of effective strength $g(1-f)$. Such partial offset of weight by buoyancy obtains, for example, for a dirigible or blimp that relies on buoyancy for only part of its lift, with the balance obtaining aerodynamically, or for a hydrofoil that cruises so slowly that it must rely on buoyancy for a ${ }^{2}$ For more technical complements to Ref. [9] see Refs. [10] [45]. 
non-negligible part of its lift.

For numerical examples, consider a $100 \mathrm{~km}=10^{5} \mathrm{~m}$ aerodynamic level flight at a given altitude $h_{A}$ (within the troposphere) with $L / D=20$ versus a $100 \mathrm{~km}=10^{5} \mathrm{~m}$ ballistic flight launched at a $45^{\circ}$ angle, beginning and ending at this same altitude $h_{B \text {,initial }}=h_{B \text {,final }}=h_{A}$. Let the mass of both the aerodynamic and ballistic craft be $1000 \mathrm{~kg}$. Assume that the engines powering both the aerodynamic and ballistic craft are $25 \%$ efficient. Neglect air resistance in the ballistic flight. Then by elementary Newtonian mechanics [1] the ballistic flight will peak at an altitude $25 \mathrm{~km}=2.5 \times 10^{4} \mathrm{~m}$ above $h_{A}$, low enough that our ballistic craft, as our aerodynamic craft, can be powered by air-breathing engines. Taking $g=9.8 \mathrm{~m} / \mathrm{s}^{2}$ and accounting for the $25 \%$ efficiency of the engines, by Equation (1), which is applicable because $100 \mathrm{~km} \ll R \doteq 6370 \mathrm{~km}$,

$$
\frac{E_{B, \text { short }}}{\epsilon}=\frac{1}{0.25} \times \frac{1}{2} m g X \mathrm{~J}=2 m g X \mathrm{~J}=2 \times 1000 \times 9.8 \times 10^{5} \mathrm{~J}=1.96 \times 10^{9} \mathrm{~J} .
$$

And by Equation (2),

$$
\frac{E_{A}}{\epsilon}=\frac{1}{0.25} \times \frac{m g X}{L / D} \mathrm{~J}=\frac{4 m g X}{20} \mathrm{~J}=\frac{1000 \times 9.8 \times 10^{5} \mathrm{~J}}{5}=1.96 \times 10^{8} \mathrm{~J} .
$$

Since typical hydrocarbon aviation fuels yield $\approx 4.4 \times 10^{7} \mathrm{~J} / \mathrm{kg},{ }^{3} \approx 45 \mathrm{~kg}$ of fuel would be required for the ballistic flight, and $\approx 4.5 \mathrm{~kg}$ of fuel would be required for the aerodynamic level flight. (This yield of $\approx 4.4 \times 10^{7} \mathrm{~J} / \mathrm{kg}$ is per $\mathrm{kg}$ of fuel alone, not counting the $\mathrm{O}_{2}$ required to burn it.) Not neglecting air resistance in the ballistic flight, the energy-efficiency advantage of the aerodynamic level flight would exceed this 10:1 ratio. Engine efficiency is discussed in more detail, and distinguished from the energy efficiency of flight per se, in Section 2.4. In any case, as per the numerical examples given immediately above, the energy that must be supplied to an engine whose efficiency is $\epsilon$ in order to facilitate aerodynamic flight requiring energy $E_{A}$ is of course $E_{A} / \epsilon$. And likewise in the case of ballistic flight it is of course $E_{B} / \epsilon$.

\subsection{Intermediate-Length Flights (Including Single Circumnavigation): Aerodynamic Flight Still Usually Wins, But It's Closer}

Now let us consider the longest possible flight whose purpose is to reach a destination on Earth-a single circumnavigation of Earth. The destination is thus the starting point after a round-the-world trip. For circumnavigation $X=2 \pi r, X$ being the horizontal distance traversed given flight at constant $h$ and hence also

\footnotetext{
${ }^{3}$ See the following article (most recently revised in 2019) at https://www.wikipedia.org: "Aviation fuel". [Note: All Wikipedia articles have Talk pages, wherein strengths and weaknesses of the articles, along with suggestions for their improvement, are discussed. Wikipedia articles are typically revised frequently. Almost all, if not all, Wikipedia articles cited in this paper have been most recently revised in 2019 as of this writing. So all Wikipedia articles cited in this paper are denoted with (most recently revised in 2019).]
} 
at constant $r=R+h$, following Earth's curvature.

In this case, by elementary Newtonian mechanics minimum-energy ballistic spaceflight obtains at the lowest-altitude circular orbit at which air resistance or drag is negligible, i.e., at $h_{B} \approx 100 \mathrm{mi} \approx 160 \mathrm{~km}$ or equivalently at $r_{B}=R+h_{B} \approx 6370 \mathrm{~km}+160 \mathrm{~km}=6530 \mathrm{~km} .{ }^{4}$ By elementary Newtonian mechanics a circular orbit requires speed $v_{\text {orbit }}=\left(G M / r_{B}\right)^{1 / 2}$, where $M$ is Earth's mass (not to be confused with the mass $m$ of a spacecraft). ${ }^{4}$ Also by elementary Newtonian mechanics the energy of a spacecraft of mass $m$ at rest at Earth's surface is its gravitational potential energy $E_{\text {pot }}(R)=-G M m / R$. Its gravitational potential energy at $r_{B}=R+h_{B}$ is $E_{\text {pot }}\left(h_{B}\right)=-G M m /\left(R+h_{B}\right)$, its orbital kinetic energy at $r_{B}=R+h_{B}$ is $E_{\text {kin,orbit }}\left(h_{B}\right)=G M m /\left[2\left(R+h_{B}\right)\right]=m v_{\text {orbit }}^{2} / 2$, and its total (potential + kinetic) energy in circular orbit at $r_{B}=R+h_{B}$ is $E_{\text {total }}\left(h_{B}\right)=-G M m /\left[2\left(R+h_{B}\right)\right] \cdot{ }^{4}$ In this Section 2.2 we consider only minimum-altitude $\left(h_{B} \approx 100 \mathrm{mi} \approx 160 \mathrm{~km}\right)$ circular spaceflight orbits for which $h_{B} \ll R$ and hence $v_{\text {orbit }}=\left(G M / r_{B}\right)^{1 / 2} \doteq(G M / R)^{1 / 2}$. If $h_{B} \ll R$, then by elementary Newtonian mechanics the total energy cost of establishing orbit and hence of ballistic circumnavigation of Earth at $r_{B}=R+h_{B} \quad$ is ${ }^{4}$

$$
\begin{aligned}
E_{\text {total }}\left(h_{B}\right) & =E_{B, \text { circumnavigation }}=-\frac{G M m}{2\left(R+h_{B}\right)}-\left(-\frac{G M m}{R}\right) \\
& =\frac{G M m}{R}-\frac{G M m}{2\left(R+h_{B}\right)}=\frac{G M m}{R}-\frac{G M m}{2 R\left(1+\frac{h_{B}}{R}\right)} \\
& \doteq \frac{G M m}{R}-\frac{G M m}{2 R}\left(1-\frac{h_{B}}{R}\right)=\frac{G M m}{R}\left[1-\frac{1}{2}\left(1-\frac{h_{B}}{R}\right)\right] \\
& =\frac{G M m}{R}\left(1-\frac{1}{2}+\frac{h_{B}}{2 R}\right)=\frac{G M m}{R}\left(\frac{1}{2}+\frac{h_{B}}{2 R}\right) \\
& =\frac{G M m}{2 R}\left(1+\frac{h_{B}}{R}\right) \doteq \frac{G M m}{2 R}=\frac{1}{2} m \times \frac{G M}{R^{2}} \times R \\
& \doteq \frac{1}{2} m g R=\frac{1}{2} \frac{m g \times 2 \pi R}{2 \pi}=\frac{m g \times 2 \pi R}{4 \pi} \doteq \frac{m g X}{4 \pi} .
\end{aligned}
$$

The first step in the last line of Equation (3) is justified because at all $r \geq R$, $g=G M / r^{2}$, and specifically at $r=R, g=G M / R^{2}$. (We are primarily interested in the magnitude of $g$, so we always take $g$ as positive.)

For all $r \geq R$ circular orbital speed at $r$ is $v_{\text {orbit }}=(G M / r)^{1 / 2}$ and escape velocity from $r$ is $v_{\text {escape }}=(2 G M / r)^{1 / 2}{ }^{4}$ Thus energy cost for escape from $r$ to infinity can be written as ${ }^{4}$

$$
\begin{aligned}
E_{\text {escape }} & =\frac{1}{2} m v_{\text {escape }}^{2}=\frac{1}{2} m \frac{2 G M}{r}=m \frac{G M}{r}=m \frac{G M}{r^{2}} r=m g r \text { at all } r \geq R \\
& =m g R \text { at } r=R .
\end{aligned}
$$

The energy cost establishing of circular orbital speed at $r$, which equals the circular-orbital kinetic energy at $r$, is half as great: ${ }^{4}$

${ }^{4}$ See also Ref. [1] Chap. 13. 


$$
\begin{aligned}
E_{\text {kin,orbit }} & =\frac{1}{2} m v_{\text {orbit }}^{2}=\frac{1}{2} m \frac{G M}{r}=\frac{1}{2} m \frac{G M}{r^{2}} r=\frac{1}{2} m g r \text { at all } r \geq R \\
& =\frac{1}{2} m g R \text { at } r=R .
\end{aligned}
$$

Thus at all $r \geq R, E_{\text {escape }}$ from radial distance $r$ from Earth's center to infinity equals the energy required for lifting through vertical distance $r$ from radial distance $r$ to radial distance $2 r$ from Earth's center if $g$ had remained constant and equal to its value at $r$ rather than decreasing with increasing distance from Earth. And $E_{\text {kin,orbit }}$ for circular orbit at $r$ is half as great. Hence also at all $r \geq R$ the free-fall velocity through vertical distance $r$ from radial distance $2 r$ to radial distance $r$ from Earth's center if $g$ had been constant and equal to its value at $r$ equals the escape velocity from radial distance $r$ from Earth's center. Thus the "gravitational scale height" at all $r \geq R$ is equal to $r$ itself-specifically, at $r=R$ it is equal to $R$ itself [16] [17] [18]. ${ }^{4}$

The " $r=0$ gravitational scale height" corresponding to escape through a borehole from the center $r=0$ of a spherical gravitator of uniform density (which Earth is not) is 3/2 times that from $R$. Hence the energy cost for escape through a borehole from the center $r=0$ of a spherical gravitator of uniform density is $3 / 2$ times that from $R$. This is perhaps most easily understood if one observes that within a uniform-density spherical gravitator, i.e., at $0 \leq r \leq R$, $g \propto r$. Therefore the average value of $g(r)$ within $R$ equals $g(R) / 2$. Thus the portion of an escape trip from $r=0$ within $R$ contributes $R / 2$ to the " $r=0$ gravitational scale height", the portion at and beyond $R$ contributes $R$ itself, total $3 R / 2$. Thus the portion of an escape trip from $r=0$ within $R$ costs $1 / 2$ as much energy (1/3 of the total) as the portion at and beyond $R(2 / 3$ of the total). Thus $E_{\text {escape }}(r=0)=\frac{3}{2} E_{\text {escape }}(r=R)$. Escape velocity from $R$ is $(2 G M / R)^{1 / 2} ;{ }^{4}$ since $v_{\text {escape }} \propto E_{\text {escape }}^{1 / 2}$ escape velocity through a borehole from the center $r=0$ of a spherical gravitator of uniform density is $v_{\text {escape }}(r=0)=\left(\frac{3}{2}\right)^{1 / 2} v_{\text {escape }}(r=R)=(3 G M / R)^{1 / 2}$.

Of course $M$ can be construedas the mass of any spherically-symmetrical gravitator for which Newtonian gravitational theory is sufficiently accurate and Einstein's General Relativity is not required, not necessarily Earth (the spherically-symmetrical gravitator taken to be necessarily of uniform density only in our discussion of $r=0$ gravitational scale height). But in this Section 2.2 our main focus is on comparison of single-circumnavigation aerodynamic flight versus single-circumnavigation minimum-altitude circular-orbit ballistic spaceflight about Earth. (For auxiliary information concerning the concept of scale height see Supplementary Note 7.)

Since even minimum-altitude circular-orbit ballistic spaceflight must be above any appreciable atmosphere, $r_{B}=R+h_{B}$ for a single-orbit spaceflight must exceed $r_{A}=R+h_{A}$ for a single-circumnavigation aerodynamic flight. But since 
for simplicity we let the spaceflight orbit be a minimum-altitude circular one, $h_{B} \ll R$ and hence slightly more strongly not only $h_{A} \ll R$ but also $h_{B}-h_{A}=r_{B}-r_{A} \ll R$. The concept of gravitational scale height introduced in the immediately preceding paragraph may help clarify why the strong inequality $h_{B} \ll R$ ensures that the result derived for $E_{\text {total }}\left(h_{B}\right)=E_{B \text {,circumnavigation }}$ in Equation (3) is sufficiently accurate for our purposes. ${ }^{4}$

Thus if $h_{B}$ is minimum-orbital altitude then $h_{B}-h_{A}=r_{B}-r_{A}$ can be neglected compared to $R$, slightly more so compared to $r_{A}=R+h_{A}$, and slightly more so yet compared to $r_{B}=R+h_{B}$. Hence with negligible error we can set $r_{A} \doteq r_{B} \doteq\left(r_{A}+r_{B}\right) / 2=r \doteq R$. Then for the longest possible (single-circumnavigation) aerodynamic level flight at altitude $h_{A}=r_{A}-R$

$\left(h_{A}<h_{B} \ll R\right.$ ) whose purpose is to reach a destination (the starting point after a round-the-world trip) on Earth, Equation (2) remains valid if we set $X=2 \pi r_{\mathrm{A}} \doteq 2 \pi R$. Hence

$$
E_{A, \text { circumnavigation }}=\frac{m g X}{L / D}=\frac{m g \times 2 \pi r_{A}}{L / D} \doteq \frac{m g \times 2 \pi R}{L / D} .
$$

Comparing Equations (3) and (6), if $L / D>4 \pi$ then

$E_{A, \text { circumnavigation }}<E_{B \text {,circumnavigation }}$. Hence single-circumnavigation aerodynamic level (fixed-altitude) flight with $L / D>4 \pi$ is more energy-efficient than single-circumnavigation minimum-altitude circular-orbit ballistic spaceflight, neglecting the very small air resistance in the latter. This requirement $L / D>4 \pi$ is met by many, perhaps most, but not all aircraft. It is met by soaring birds such as albatrosses [2]-[15]. It is very easily met by sailplanes [2]-[15]. Thus the longest possible (single-circumnavigation) aerodynamic level flight whose purpose is to reach a destination (the starting point after a round-the world trip) on Earth is in many, perhaps most, but not all cases more energy-efficient than the corresponding (single-circumnavigation) minimum-altitude circular-orbit ballistic spaceflight. This requirement $L / D>4 \pi$ neglects air resistance in minimum-altitude circular-orbit ballistic spaceflight; if it is not neglected, then the requirement is weakened to $L / D>4 \pi n$ with $n<1$. But it is weakened only very slightly, because air resistance even at minimum circular-orbit spaceflight altitude is very small. (Since if $X=2 \pi R$ minimum-energy ballistic spaceflight is a circular orbit just above appreciable atmosphere at altitude $h_{B}$, it cannot begin and end at the altitude $h_{A}$ of aerodynamic level flight, but $h_{B}-h_{A} \ll R$.)

Neglecting air resistance in minimum-altitude ballistic spaceflight, for flights of intermediate length ( $X$ ranging from much smaller than $R$ to approaching $2 \pi R)$, the minimum value that $L / D$ must exceed for aerodynamic level flight at altitude $h_{A} \ll R$ to be more energy-efficient than ballistic flight beginning and ending at this same altitude $h_{B, \text { initial }}=h_{B, \text { final }}=h_{A}$ increases monotonically from 2 towards $4 \pi$ as $X$ increases from very small values towards $2 \pi R$. Not neglecting air resistance in ballistic flight, for flights of intermediate length ( $X$ ranging from much smaller than $R$ to approaching $2 \pi R$ ), the minimum value that $L / D$ must exceed for aerodynamic level flight at altitude $h_{A} \ll R$ to be 
more energy-efficient than ballistic flight beginning and ending at this same altitude $h_{B \text {,initial }}=h_{B \text {, final }}=h_{A}$ increases monotonically from $2 n(X)$ towards $4 \pi n(X)$ as $X$ increases from very small values towards $2 \pi R: n(X)<1$ but increases monotonically towards very nearly 1 as $X$ increases from very small values towards $2 \pi R$. Air resistance is very small in minimum-altitude circular-orbit ballistic flight ( $X=2 \pi R)$, and hence also $1-n(X)$ is very small, i.e., $n(X)$ is very nearly 1 if $X=2 \pi R$. (Since if $X=2 \pi R$ minimum-energy ballistic spaceflight is a circular orbit just above appreciable atmosphere at altitude $h_{B}$, it cannot begin and end at the altitude $h_{A}$ of aerodynamic level flight, but $\left.h_{B}-h_{A} \ll R.\right)$

Generalizing the third-to-last paragraph of Section 2.1 in light of this Section 2.2, by Equations (1)-(3) and (6), if air resistance in ballistic flight can be neglected, then for flights traversing any given horizontal distance $X$, short or long, both $E_{B}$ and $E_{A}$ are directly proportional to $\mathrm{mg}$. (Of course, a long horizontal flight follows Earth's curvature at fixed altitude above mean sea level.) Hence reducing $m g$ reduces the energy cost of both ballistic flight and aerodynamic level flight traversing any given horizontal distance $X$, short or long, equally and in direct proportion to the reduction in $\mathrm{mg}$, but does not alter the ratio of energy costs between these two modes of flight. If air resistance in ballistic flight cannot be neglected, then reducing $m g$ reduces the energy cost of any ballistic flight, short or long, less than in direct proportion to the reduction in $\mathrm{mg}$. (The second-to-last paragraph of Section 2.1 requires no modification in light of Section 2.2.)

\subsection{Long Flights (Multicircumnavigation and Beyond): Ballistic Flight Wins-Big-Time}

For an $N$-circular-orbit minimum-altitude ballistic spaceflight traversing distance $X=2 \pi r_{B} N=2 \pi\left(R+h_{B}\right) N \doteq 2 \pi R N$, whose purpose is typically scientific study of Earth as opposed to reaching a destination on Earth, in almost all cases $N \gg 1$. Also $X=2 \pi r_{A} N=2 \pi\left(R+h_{A}\right) N \doteq 2 \pi R N$ for $N$-circumnavigation aerodynamic level (fixed-altitude) flight. By Equation (2), for $N$-circumnavigation aerodynamic level (fixed-altitude) flight, $E_{A}$ increases linearly with $X=2 \pi r_{A} N=2 \pi\left(R+h_{A}\right) N \doteq 2 \pi R N$. By contrast, for minimum-altitude circular-orbit ballistic spaceflight, irrespective of $X=2 \pi r_{B} N=2 \pi\left(R+h_{B}\right) N \doteq 2 \pi R N, E_{B}$ remains fixed at the value given by Equation (3) for $N=1$. For, even at minimum-orbital spaceflight altitude, air resistance is almost negligible, i.e., space is almost frictionless; thus the energy cost of launching a spacecraft is one-time. Hence for flights traversing $N$ circumnavigations of Earth $L / D$ must exceed $4 \pi N$ if aerodynamic level (fixed-altitude) flight is to be more energy-efficient than minimum-altitude circular-orbit ballistic spaceflight. But for even the best currently existing sailplanes $L / D$ values are between 70 and 80 [2]-[15], and $L / D \approx 100$ may represent the practicable limit that can be achieved even with the most advanced aerodynamic technology [2]-[15]. Hence even minimum-altitude $\left(h_{B} \ll R\right)$ ballistic $N$-circular-orbit $(N \gg 1$ ) spaceflight about Earth is incomparably more ener- 
gy-efficient than $N$-circumnavigation aerodynamic level (fixed-altitude) flight within Earth's atmosphere. This is even more strongly true with respect to high-altitude spaceflight for which air resistance is even more negligible, even with the one-time energy cost of launch being larger than for minimum-altitude circular-orbit spaceflight (recall Section 2.2). ${ }^{4}$ In return for a one-time energy expenditure, $X_{\text {spaceflight }} / 2 \pi R \rightarrow \infty$ even for minimum-altitude circular-orbit spaceflight, $X_{\text {spaceflight }} / 2 \pi R \rightarrow \infty$ even more strongly for high-altitude, say geosynchronous, circular-orbital spaceflight, and $X_{\text {spaceflight }} / 2 \pi R \rightarrow \infty$ even more strongly yet for spaceflight exceeding escape velocity. This of course is simply owing to space being essentially frictionless, and increasingly frictionless with increasing altitude, thus allowing spacecraft but not aircraft to take full advantage of Newton's first law of motion (inertia). ${ }^{5}$ The energy cost of speed in spaceflight is one-time, the energy cost of speed in aerodynamic flight is never-ending. ${ }^{5}$ Spaceflight is thus the only mode of transportation that can achieve $\infty \mathrm{mi} / \mathrm{gal}=\infty \mathrm{km} / \mathrm{l}$ of fuel (or the equivalent thereof) - Spaceship Earth (whose fuel for its orbital and rotational motions was part of the solar nebula's kinetic energy) is a good example. ${ }^{5}$ To save time in spaceflight continuous energy expenditure can be employed, for example employing solar, laser, or on-board nuclear energy. But in spaceflight continuous energy expenditure buys acceleration; in aerodynamic flight it buys only (constant) speed. ${ }^{5}$

\subsection{Flight Energy Efficiency versus Engine Energy Efficiency}

The energy efficiency of flight per se should not be confused with the energy efficiency of the engine that powers flight. If an aircraft engine is a heat engine operating in a cycle with heat input at temperature $T_{\text {hot }}$ and heat exhaust at temperature $T_{\text {cold }}$, then its thermodynamic efficiency even assuming perfect reversibility is limited by the Carnot bound $1-\left(T_{\text {cold }} / T_{\text {hot }}\right) .{ }^{6}$ Of course any real cyclic heat engine is less than perfect and hence its actual thermodynamic efficiency is less than the Carnot $^{6}$ bound. But the actual thermodynamic efficiency of any real cyclic heat engine under any given conditions, while less than the Carnot bound $1-\left(T_{\text {cold }} / T_{\text {hot }}\right)$ for any given $T_{\text {cold }} / T_{\text {hot }}$, nonetheless, all other things being equal, like the Carnot $^{6}$ bound increases monotonically with decreasing $T_{\text {cold }} / T_{\text {hot }}$. For example, the Curzon-Ahlborn efficiency at maximum power output assuming endoreversibility (irreversible heat flows directly proportional to finite temperature differences but otherwise reversible operation), $1-\left(T_{\text {cold }} / T_{\text {hot }}\right)^{1 / 2}$ [19] [20], ${ }^{7}$ like the Carnot efficiency $1-\left(T_{\text {cold }} / T_{\text {hot }}\right),{ }^{6}$ increases monotonically with decreasing $T_{\text {cold }} / T_{\text {hot }}$ [19] [20]., ${ }^{6,7}$ Both the Carnot and Curzon-Ahlborn [19] [20] efficiencies are upper bounds. ${ }^{6,7}$ Unlike the Carnot efficiency, the Curzon-Ahlborn [19] [20] efficiency does not neglect irreversible heat flows (assumed directly proportional to finite temperature differences), but both efficiencies neglect all other losses, e.g., friction, energy wasted as 
sound, etc. (While Curzon and Ahlborn derived the Curzon-Ahlborn efficiency independently [19], it had been derived previously [20].7) Thus if an aircraft engine is a cyclic heat engine, then this engine will operate most efficiently at the altitude where the atmosphere is coldest, most typically at or near the tropopause, but commonly as close to the surface as is safe in polar regions in winter. This of course assumes that the engine, if air-breathing and operating at altitude, is supercharged, and that the supercharger requires only a very small fraction of the engine's power output. The oxygen available to an air-breathing engine is of course directly proportional to air density $\rho$, so intuitively it would seem that so would be the engine's power output. But actually with increasing altitude $h_{A}$ the power output of an unsupercharged air-breathing engine decreases slightly faster than $\rho .{ }^{8}$ Even a decrease in power output directly proportional to $\rho$ in almost all cases more than offsets any increase in power output owing to increased thermodynamic efficiency on account of lower temperatures that typically obtain at higher altitudes. ${ }^{6,7}$ (A real cyclic heat engine may be difficult to start in cold weather, and its efficiency immediately at starting may be reduced by the high viscosity of still-cold lubricants, but upon attaining steady-state it will operate more efficiently than in hot weather.) In any case, the energy that must be supplied to an engine whose efficiency is $\epsilon$ in order to facilitate aerodynamic flight requiring energy $E_{A}$ is of course $E_{A} / \epsilon$. And likewise in the case of ballistic flight it is of course $E_{B} / \epsilon$.

Of course, neither nonheat engines such as electric motors and birds' flight muscles nor noncyclic (necessarily single-use) heat engines such as rockets are limited ultimately by the Carnot bound, nor are they limited at maximum power output assuming endoreversibility by the Curzon-Ahlborn bound. (Even if a rocket engine is refurbished, each launch represents a separate single use.) Their Carnot efficiencies and even their Curzon-Ahlborn efficiencies can in principle approach $100 \%$ irrespective of $T_{\text {cold }} / T_{\text {hot }}$ and indeed of temperature at all. But, more often than not, in practice these engines face other limitations. Electric motors typically in practice rather than merely in principle exceed $90 \%$ efficiency. But birds' flight muscles are in practice typically considerably less efficient, typically in the range of $25 \%$ to $40 \%$. And even if noncyclic, single-use rocket heat engines can in practice rather than merely in principle approach $100 \%$ efficiency irrespective of $T_{\text {cold }} / T_{\text {hot }}$ and indeed of temperature at all, typically most of their work output must be expended in accelerating exhaust gases, with only a small fraction available for accelerating payloads. ${ }^{9}$

We should note that: (a) Even if rocket heat engines operated in a cycle, owing ${ }^{8}$ See Ref. [10], Sections XIII.1-XIII.2 and Chap. XIV (in Chap. XIV see especially pp. 389-394 and Sections XIV.4-XIV.5); also Ref. [13], pp. 19-20 and Chaps. VI-IX (especially Chap. VI and pp. 69-70, 76-78, and 80-81; most especially compare unsupercharged engine power versus air density as a function of altitude via Table III on p.19 and Figure 37 on p. 63).

${ }^{9}$ Information concerning spacecraft propulsion is provided in the following articles (all most recently revised in 2019) at https://www.wikipedia.org: "Tsiolkovsky rocket equation", "Spacecraft propulsion", "Rocket engine”, Rocket propellant, "Rocket”, "Specific Impulse”, "Electrically powered spacecraft propulsion", "Liquid rocket propellant", "Solar sail”, "Electric sail”, "Magnetic sail”, and "RF resonant cavity thruster". See also: Scoles, S. (August 2019) The Good Kind of Crazy. Sci. Am. $321(2), 58-65$. 
to their very small $T_{\text {cold }} / T_{\text {hot }}$ ratio their Carnot and even Curzon-Ahlborn bound would be nearly $100 \%$. But it would still obtain that typically most of their work output must be expended in accelerating exhaust gases, with only a small fraction available for accelerating payloads. ${ }^{9}$ (b) Not all rocket engines are heat engines, not even noncyclic ones. For example, ion-drive rocket engines are not heat engines, not even noncyclic ones, and hence (like noncyclic heat engines) are not limited ultimately by the Carnot bound, nor at maximum power output assuming endoreversibility by the Curzon-Ahlborn bound. Because of their high exhaust speeds less mass need be exhausted to achieve a given spacecraft speed, and hence less of their work output need be expended on the exhaust. ${ }^{9}$ (c) Nonrocket spacecraft propulsion, ${ }^{9}$ e.g., via solar sails or laser sails, or via the EM, MEGA or related drives if they are verified, ${ }^{9}$ is not limited ultimately by the Carnot bound, nor at maximum power output assuming endoreversibility by the Curzon-Ahlborn bound.

As an aside, we mention that the ratio of the Curzon-Ahlborn efficiency to the Carnot efficiency, $\left[1-\left(T_{\text {cold }} / T_{\text {hot }}\right)^{1 / 2}\right] \div\left[1-\left(T_{\text {cold }} / T_{\text {hot }}\right)\right]$, decreases monotonically with increasing $T_{\text {cold }} / T_{\text {hot }}$ from unity in the limit $T_{\text {cold }} / T_{\text {hot }} \rightarrow 0$ to $1 / 2$ in the limit $T_{\text {cold }} / T_{\text {hot }} \rightarrow 1$. Thus this ratio is never greater than 1 or less than $1 / 2$. The former limit is obvious. The latter limit is most easily verified by setting $T_{\text {cold }} / T_{\text {hot }}=1-\delta:$ in the limit $T_{\text {cold }} / T_{\text {hot }} \rightarrow 1 \Leftrightarrow \delta \rightarrow 0$ with the help of the binomial theorem $\left[1-(1-\delta)^{1 / 2}\right] \div[1-(1-\delta)]=\left[1-\left(1-\frac{1}{2} \delta\right)\right] \div \delta=\frac{1}{2} \delta \div \delta=\frac{1}{2}$.

Of course, determination of how closely any given engine approaches to its theoretical maximum efficiency requires detailed analyses of the properties of that particular engine (e.g., bypass ratio of jet engines, battery and circuit design for electric motors, metabolic chemistry of birds' flight muscles, etc.) We have not considered such detailed analyses in this Section 2.4 .

\section{Air Density and Aerodynamic Level Flight}

\subsection{A Simplified View of Drag and Lift}

\subsubsection{The Paramount $\rho v^{2}$ Functional Dependency}

Drag is given by [2]-[15]

$$
D=\frac{1}{2} C_{D} A_{\text {frontal,geom }} \rho v^{2}=\frac{1}{2} A_{\text {frontal, eff }} \rho v^{2},
$$

where $C_{D}$ is the coefficient of drag, $A_{\text {frontal,geom }}$ is an aircraft's geometrical frontal cross-sectional area, $A_{\text {frontal,eff }}$ is its effective frontal cross-sectional area (the frontal cross-sectional area that it effectively presents with respect to air resistance or drag), $\rho$ is the air density, and $v$ is the airspeed (also the ground speed if the wind is calm) of flight. [Note: The symbol $A$ denoting surface area should not be confused with the subscript $A$, introduced in the first paragraph of Section 2.1, denoting aerodynamic flight (as opposed to ballistic flight, denoted by the subscript $B$ ).] Thus the coefficient of drag $C_{D}$ is given by

$$
C_{D}=\frac{A_{\text {frontal,eff }}}{A_{\text {frontal,geom }}} .
$$


(See Supplementary Note 8.)

But more complete definitions of $A_{\text {frontal,eff }}$ and hence of $C_{D}$ are required. The drag owing to an aircraft's frontal cross-sectional area per se is pressure drag. Pressure drag times frontal cross-sectional area is the force that an aircraft must impart to push air in front out of its way, and to overcome the pull of the partial vacuum behind it ensuing because adjacent air cannot move in behind it instantaneously. But there are two other components of drag: induced drag-a penalty that must be paid for lift (see Supplementary Notes 6 and 9), and skin-friction drag-owing to the viscosity of air rubbing against surfaces parallel to the airflow (see Supplementary Note 10). The contributions of induced drag and skin-friction drag are included along with that of pressure drag within $A_{\text {frontal,eff }}$ and hence within $C_{D}$. Thus our more complete definitions of $A_{\text {frontal,eff }}$ and hence of $C_{D}: A_{\text {frontal,eff }}$ is an aircraft's effective frontal cross-sectional area-the frontal cross-sectional area that it would effectively present with respect to total air resistance or drag - as if total drag had been subsumed within pressure drag, i.e., as if induced drag and skin-friction drag had been converted to and incorporated within pressure drag. Since $A_{\text {frontal,geom }}$ is fixed for any given aircraft flying at any given angle of attack, by Equations (7) and (8) thus construing induced drag and skin-friction drag as incorporated within pressure drag modifies $C_{D}$ in direct proportion to the modification of $A_{\text {frontal,eff }}$ from its value with respect to pressure drag alone. Accordingly, we thus construe Equations (7) and (8) as if induced drag and skin-friction drag are converted to and incorporated within pressure drag. Drag is a complex phenomenon [2]-[15]. Other classifications of drag are sometimes used. (See Supplementary Note $8 .{ }^{10}$ ) But regardless of classification scheme we construe $A_{\text {frontal, eff }}$ and hence $C_{D}$ as noted immediately above, i.e., as if all drag is subsumed within pressure drag. This implies subsuming all of the complexities within $C_{D}$ : with that understood, Equations (7) and (8) are correct as written. With good aerodynamic design, at or near the angle of attack at which $L / D$ is maximized, $C_{D} \ll 1$ and hence $A_{\text {frontal,eff }}=C_{D} A_{\text {frontal,geom }} \ll A_{\text {frontal,geom }}$ (see Supplementary Notes 4 and 5). (In the case of hovering flight, as of a helicopter, or of a hovering hummingbird or insect, even if the aircraft executes no horizontal motion the revolving airfoils do and hence experience drag. The revolving airfoils' geometrical area $A_{\text {frontal,geom }}$ with respect to drag is their geometrical frontal cross-sectional area, their effective area $A_{\text {frontal,eff }}$ with respect to drag is their effective frontal cross-sectional area; $V$ with respect to drag is the root-meansquare average, taken over the geometrical frontal cross-sectional area of the airfoils, of airspeeds of the airfoils, be they blades of a helicopter, or wings of a hovering hummingbird or insect. Of course, if a hovering-flight aircraft also executes horizontal motion then its entire structure contributes to both $A_{\text {frontal,geom }}$ ${ }^{10}$ See the following articles: "Drag (physics)", "Drag equation", and "Drag coefficient" (all most recently revised in 2019) at https://www.wikipedia.org, and other Wikipedia articles concerning drag that are cited therein. See also "Drag" (most recently revised in 2017) and "AP4ATCO - Drag - Types and Effects" (most recently revised in 2015) at https://www.skybrary.aero/index.php/Main Page, and other SKYbrary articles concerning drag that are cited therein. 
and $\left.A_{\text {frontal,eff }}.\right)$

Similarly (see Supplementary Notes 1-6 and 8), lift [2]-[15] is given by

$$
L=m g=\frac{1}{2} C_{L} A_{\text {wing,geom }} \rho v^{2}=\frac{1}{2} A_{\text {wing,eff }} \rho v^{2},
$$

where $C_{L}$ is the coefficient of lift, $A_{\text {wing,geom }}$ is an aircraft's geometrical wing area, and $A_{\text {wing,eff }}$ is its effective wing area-the area that it effectively presents with respect to downward deflection of air required by Newton's third law of motion ${ }^{11}$ as the price for the upward force that is its lift. The first step of Equation (9) recognizes that to maintain aerodynamic level flight $L$ must equal the weight $m g$ of an aircraft. We use "wing area" as shorthand for an aircraft's entire lifting-surface area. A typical airplane obtains most but not all of its lift from its wings; its fuselage and elevators contribute some lift. The spectrum of airplane design ranges from flying wings with little or no fuselage to lifting bodies that are fuselage with little or no wing. ${ }^{12}$ Flying wings are based on the principle that the wing has a higher $L / D$ ratio than any other part of an airplane, while lifting bodies seek to avoid structural stresses on wings, especially at high airspeeds. ${ }^{12}$ Thus the coefficient of lift $C_{L}$ is given by

$$
C_{L}=\frac{A_{\text {wing,eff }}}{A_{\text {wing,geom }}} .
$$

Lift is a complex phenomenon, perhaps even more so than drag [2]-[15]. There seems to be no universal agreement concerning which explanation or combinations of explanations of lift is most correct [2]-[15]. (See also Supplementary Notes 1-6.) Equations (9) and (10) thus imply subsuming all of the complexities within $C_{L}$. But irrespective of the complexities, the bottom line is that Newton's third law of motion ${ }^{11}$ must be obeyed. With that understood, Equations (9) and (10) are correct as written. (There does seem to be universal agreement that some elements of explanations of lift are incorrect, e.g., the "equal-transit-time" element: see Supplementary Note 1.) With good aerodynamic design, at or near the angle of attack at which $L / D$ is maximized, $C_{L}$ is usually at least a significant fraction of unity and in some cases only a little smaller than unity, and hence $A_{\text {wing,eff }}=C_{L} A_{\text {wing,geom }}$ is usually at least a significant fraction of $A_{\text {wing,geom }}$ and in some cases almost as large as $A_{\text {wing,geom }}$ (see Supplementary Notes 4 and 5). At larger angles of attack $C_{L}$ not uncommonly exceeds unity, but at the expense of smaller $L / D$ (see the last four paragraphs of Section 3.1.2 and Supplementary Notes 6 and 9). [In the case of hovering flight, as of a helicopter, or of a hovering hummingbird or insect, the wings are

\footnotetext{
${ }^{11}$ See Sect. 5-3 of Ref. [1] for general discussions concerning Newton's third law of motion. For perhaps the most concise explanations pertaining to Newton's third law of motion as the bottom line with respect to aerodynamic lift, see pp. 27-29 of Ref. [13] and Section 3.1 of Decker, J.S. (2014) See How It Flies. https://www.av8n.com/how/. See also pp. 305-306 of Ref. [3] and Supplementary Notes 1 and 2.

${ }^{12}$ See the following articles (all most recently revised in 2019) at https://www.wikipedia.org: "Flying wing", "List of flying wings", and "Lifting body".
} 
the revolving airfoils. The revolving airfoils' geometrical area with respect to lift is their geometrical wing (not frontal) area, their effective area with respect to lift is their effective wing (not frontal) area; $V$ with respect to lift is the root-mean-square average, taken over the geometrical wing area of the airfoils, of airspeeds of the airfoils, be they blades of a helicopter, or wings of a hovering hummingbird or insect.]

The forms of Equations (7)-(10), in particular the $\rho v^{2}$ functional dependency in Equations (7) and (9) that is paramount for both drag and lift, can perhaps in some measure be most easily physically understood via the following very simplified qualitative to semiquantitative arguments. First, note that both drag and lift are forces, and that $\rho v^{2} A$ is the only combination of $\rho, v$, and $A$ that has dimensions of force, or equivalently that $\rho v^{2}$ is the only combination of $\rho$ and $v$ that has dimensions of force per unit area (= pressure). Construe, as discussed three paragraphs previously, induced drag and skin-friction drag as converted to and incorporated within pressure drag, so that total drag is construed as pressure drag. Pressure drag times effective frontal cross-sectional area $A_{\text {frontal,eff }}$ in aerodynamic level flight is the horizontal force-the horizontal momentum per unit time $t$-that an aircraft must impart to push air in front out of its way, and to pull air into the trailing partial vacuum behind it. Per unit time $t$ a volume of air $\sim v t A_{\text {frontal,eff }}$ and hence a mass of air $\sim \rho v t A_{\text {frontal,eff }}$ must thus be given speed typically comparable to $v$, but at any rate at least approximately proportional to $v$. Thus to maintain constant horizontal forward airspeed $v$ in the face of drag $D$ an aircraft must impart to air horizontal force equal to $D$, in accordance with ${ }^{11}$

$$
\begin{aligned}
D & =\text { horizontal force imparted to air } \\
& =\text { horizontal momentum imparted to air per unit time } t \\
& \sim \frac{(\text { mass of air given speed } \sim v \text { per unit time } t) \times v}{t} \\
& \sim \frac{\left(\rho v t A_{\text {frontal,eff }}\right) \times v}{t}=A_{\text {frontal,eff }} \rho v^{2} \sim C_{D} A_{\text {frontal,geom }} \rho v^{2} .
\end{aligned}
$$

The upward force of lift $L$ is by Newton's third law of motion ${ }^{11}$ equal to the downward force-the downward momentum per unit time $t$ - that an aircraft's wings must impart to air. By similar reasoning as we employed with respect to drag, the wings must impart to air downward force equal to $L$, in accordance with $^{11}$

$$
\begin{aligned}
L & =m g=\text { downward force imparted to air } \\
& =\text { downward momentum imparted to air per unit time } t \\
& \sim \frac{(\text { mass of air impelled downward per unit time } t) \times v}{t} \\
& \sim \frac{\left(\rho v t A_{\text {wing,eff }}\right) \times v}{t}=A_{\text {wing,eff }} \rho v^{2} \sim C_{L} A_{\text {wing,geom }} \rho v^{2} .
\end{aligned}
$$

The first step of Equation (12) recognizes that to maintain aerodynamic level 
flight $L$ must equal the weight $m g$ of an aircraft [2]-[15]. Note that the first two lines of Equations (11) and (12) are, essentially, statements of Newton's third law of motion ${ }^{11}$, and hence are rigorously correct. Approximations are employed only in the last two lines of Equations (11) and (12).

If $C_{L}$ is independent of $\rho$ and of $V$, as is typically approximately true at or near the most energy-efficient angle of attack and hence at or near airspeeds $V$ corresponding to maximization of $L / D$, then induced drag, as pressure drag, is approximately proportional to $\rho v^{2}$, and hence can be incorporated within Equations (7) and (8) via a simple approximately additive contribution to $C_{D}$ (see Supplementary Notes 4, 5, 6, and 9, especially Supplementary Note 9). But skin-friction drag is in general not even approximately proportional to $\rho v^{2}$ : skin-friction drag is a function of the coefficient of viscosity $\mu$ as well as of $\rho, v$, and $A$, and hence has dimensions of force even though it is not expressible as the combination $\rho v^{2} A$, or equivalently dimensions of force per unit area (= pressure) even though per unit area it is not expressible as the combination $\rho v^{2}$ (see Supplementary Note 10). But skin-friction drag is nevertheless typically incorporated within Equations (7) and (8) via a contribution to the functional dependence of $C_{D}$ on $\rho$ and on $v$. This is in accordance with Equations (7) and (8) being construed as if both induced drag and skin-friction drag are converted to and incorporated within pressure drag, as discussed in the second paragraph of this Section 3.1.1.

Thus for both drag and lift the $\rho v^{2}$ functional dependency is paramount [2]-[15], because $C_{D}, C_{L}$, and also the maximum value of the ratio $C_{L} / C_{D}$ corresponding to the most energy-efficient angle of attack are typically slowly varying functions of $\rho$ (and hence of aerodynamic flight altitude $h_{A}$ ) and of $V$ [2]-[15]. Thus by Equations (2) and (7)-(10) the maximum value of $L / D$ is typically at least approximately independent of air density $\rho$ (and hence of $h_{A}$ ) and of $v$. Hence by Equation (2) so is the minimum energy $E_{A}$ required for an aerodynamic level flight traversing given horizontal distance $X$. Thus since given aerodynamic level flight the maximum value of $L / D$ is approximately independent of $\rho$ and $L=m g$ must be maintained strictly independent of $\rho$, by Equation (2) $D$ is at least approximately independent of $\rho$ (and hence of $h_{A}$ ): higher airspeed $v$ required to maintain $L=m g$ despite smaller $\rho$ increases $D$ as much as smaller $\rho$ itself decreases $D$.

But this minimum energy $E_{A}$ must be expended faster and hence the power $P_{A}$ required for maximally energy-efficient aerodynamic level flight increases with decreasing $\rho$ (and hence with increasing $h_{A}$ ), because it is necessary to fly at faster $v$ in thinner air to maintain $L$ equal to the weight $m g$ of an aircraft and hence to maintain aerodynamic level flight despite smaller $\rho$. By differentiating Equation (2) with respect to time $t$ [or simply dividing Equation (2) by $t$ given steady aerodynamic level flight], we obtain, for the power $P_{\mathrm{A}}$ required to maintain aerodynamic level flight at speed $v$ of an aircraft of mass $m$ and weight $m g$ subject to aerodynamic lift $L$ and aerodynamic drag $D$. 


$$
\begin{aligned}
P_{A} & =\frac{\partial E_{A}}{\partial t}=D \frac{\partial X}{\partial t}=D v=\frac{D}{L} L v=\frac{D}{L} m g v=\frac{m g v}{L / D} \text { instantaneously } \\
& =\frac{E_{A}}{t}=\frac{D X}{t}=D v=\frac{D}{L} L v=\frac{D}{L} m g v=\frac{m g v}{L / D} \text { given steady flight. }
\end{aligned}
$$

The fifth steps of both lines of Equation (13) recognize that to maintain aerodynamic level flight $L$ must equal the weight $m g$ of an aircraft [2]-[15]. Note that $P_{A}$ is the power required for aerodynamic level flight, not to be confused with the power available. ${ }^{13}$ Obviously aerodynamic level flight is possible if and only if the maximum available power exceeds, or at the very least equals, the required power $P_{A} \cdot{ }^{13}$ Minimum required power $P_{A}=E_{A} / t$ allows an aircraft to maintain aerodynamic level flight for the maximum possible time (maximum endurance); maximum energy efficiency $E_{A} / X=P_{A} / v$ (which we always assume in this paper unless otherwise noted) allows an aircraft executing aerodynamic level flight to traverse the maximum possible distance. (See Supplementary Notes 5 and 6 , and the references cited therein.)

Because it is necessary to fly at faster $v$ in thinner air to maintain $L$ equal to the weight $m g$ of an aircraft and hence to maintain aerodynamic level flight despite smaller $\rho$, an aerodynamic level flight traversing given horizontal distance $X$ requires less time $t=X / v$ in thinner air, so the energy cost $E_{A}=P_{A} t=P_{A} X / v$ of aerodynamic level flight traversing given $X$, or equivalently $E_{A}$ per given $X$, i.e., $E_{A} / X=P_{A} / v$, is at least approximately independent of $\rho$ (and hence of $h_{A}$ ). Focusing on the paramount $\rho v^{2}$ functional dependency of aerodynamic lift and drag [2]-[15], by Equation (9) the airspeed $v$ required for aerodynamic lift $L$ to equal the weight $m g$ of an aircraft and hence for maintenance of aerodynamic level flight is proportional to $\rho^{-1 / 2}$. Thus by Equations (2), (7), and (9) $D \propto \rho v^{2}$ is independent of $\rho$ (and hence of $h_{A}$ ) if $L$ is to equal the weight $m g$ of an aircraft: higher $v^{2}$ required for $L$ to equal $m g$ despite smaller $\rho$ increases $D$ as much as smaller $\rho$ itself decreases $D$. Hence to maintain aerodynamic level flight the required power $P_{A}$, as the required airspeed $V$, is proportional to $\rho^{-1 / 2}: P_{A}=D v \propto \rho v^{3} \propto \rho\left(\rho^{-1 / 2}\right)^{3}=\rho^{-1 / 2}$. But flight time $t=X / v \propto X / \rho^{-1 / 2} \propto \rho^{1 / 2}$. Therefore in accordance with Equations (2) and (13) $E_{A}=P_{A} t=P_{A} X / v$ and thus $E_{A} / X=P_{A} / v$ is independent of $\rho$ (and hence of $h_{A}$ ). Thus, focusing on the paramount $\rho v^{2}$ functional dependency of aerodynamic lift and drag [2]-[15], the maximum value of $C_{L} / C_{D}$ and thus of $L / D$, and hence by Equation (2) the minimum energy cost or equivalently the maximum energy efficiency of aerodynamic level flight, is approximately independent of $\rho$ (and hence of $h_{A}$ ). Thus if $v$ is the airspeed of aerodynamic level flight required for $L$ to equal the weight $m g$ of an aircraft given air density $\rho$, then $\rho v^{2}$ is approximately a conserved quantity, approximatelyindependent of $\rho$ (and hence of $h_{A}$ ).

In the four immediately preceding paragraphs we did not explicitly consider the effects of changing mg. But (recall the third-to-last and second-to-last paragraphs ${ }^{13}$ See Ref. [10], Chaps. XIV-XVI; and Ref. [13], pp. 69-70 and Chaps. VIII, IX, and XII. 
of Section 2.1 and the last paragraph of Section 2.2) this should be explicitly considered. Irrespective of the value of $m g$, it is still true, in accordance with Equation (2), that $E_{A}=D X=m g X \div(L / D)$, and in accordance with Equation (13), that $P_{A}=E_{A} / t=m g v \div(L / D)$. Thus, all other things being equal, $E_{A}$ is directly proportional to $\mathrm{mg}$. But, all other things being equal, $P_{A}$ is not directly proportional to $\mathrm{mg}$, because the airspeed $v$ required to maintain aerodynamic level flight increases with increasing $m g$. By Equations (9), (12), and (13) we have for aerodynamic level flight

$$
\begin{aligned}
& \rho v^{2} \propto m g \Rightarrow v \propto\left(\frac{m g}{\rho}\right)^{1 / 2} \\
& \Rightarrow P_{A}=\frac{m g v}{L / D} \propto \frac{m g\left(\frac{m g}{\rho}\right)^{1 / 2}}{L / D}=\frac{(m g)^{3 / 2}}{\rho^{1 / 2}(L / D)} .
\end{aligned}
$$

We note that Equation (14) is consistent with $E_{A}$ being directly proportional to $m g$ in accordance with Equation (2). For in accordance with Equation (14) $E_{A}=P_{A} t=P_{A} X / v=m g X \div(L / D)$, in agreement with Equation (2).

\subsubsection{Exceptions to the Paramount $\rho v^{2}$ Functional Dependency}

Lift and drag are in general not exactly proportional to $\rho v^{2}$, because $C_{L}$ and $C_{D}$ are in general not strictly constant [2]-[15]. Induced drag, if not exactly proportional to $\rho v^{2}$, and skin-friction drag, which in general is not even approximately proportional to $\rho v^{2}$, contribute to nonconstancy of $C_{D}$ and hence of $C_{L} / C_{D}$. [Induced drag, like pressure drag, is typically approximately proportional to $\rho v^{2}$. But skin-friction drag is in general not even approximately proportional to $\rho v^{2}$ : skin-friction drag is a function of the coefficient of viscosity $\mu$ as well as of $\rho, V$, and $A$, and hence has dimensions of force even though it is not expressible as the combination $\rho v^{2} A$, or equivalently dimensions of force per unit area (= pressure) even though per unit area it is not expressible as the combination $\rho v^{2}$. (See Supplementary Notes 4, 5, 6, 9, and 10.) But again at or near the most energy-efficient angle of attack and thus at airspeeds $v$ at or near that corresponding to maximization of $C_{L} / C_{D}$ and hence of $L / D$, typically these contributions to nonconstancy are small. Also for pressure drag the proportionality to $\rho v^{2}$ is usually approximate rather than exact. Thus in general $C_{L}$ and $C_{D}$ vary with $\rho$ (and hence with $h_{A}$ ) and with $v$, and hence with Reynolds number [2]-[15] and with Mach number [2]-[15], and furthermore in general not at exactly the same rate, so also their ratio $C_{L} / C_{D}$ is not strictly constant [2]-[15]. In general these variations are typically small compared with the paramount $\rho v^{2}$ functional dependency (see Supplementary Notes 4, 5, 6, 9, and 10). But three major exceptions, wherein there are large departures from constancy of $C_{L}, C_{D}$, and in some cases also $C_{L} / C_{D}$, should be noted: (a) For aircraft, especially those with short wingspans, trying to maximize $L$ at the slowest possible $v$ well below that corresponding to maximization of $L / D$ by trying to maximize $C_{L}$ whatever the cost in $C_{D}$, for example in trying to 
land at the slowest possible $v$, induced drag is likely the largest component of the total drag $D$. In such cases, owing to large induced drag, total $\operatorname{drag} D$ is likely considerably larger than would typically be expected for given $\rho v^{2}$ (see Supplementary Notes 6 and 9). In regards to reducing induced drag, we should mention wingtip devices [21] [22] (see also the second paragraph of Supplementary Note 9). (b) As $v$ increases through the typically small range of values corresponding to the transition from laminar to turbulent flow, the onset of turbulence helps to fill the trailing partial vacuum, thereby greatly reducing pressure drag and hence $C_{D}$. This decrease in $C_{D}$ owing to the reduction in pressure drag is often sufficient to more than offset not only the increase in $C_{D}$ owing to the onset of turbulence itself, but over this typically small range of values of $v$ also the increase in pressure drag and indeed in total drag $D$ proportional to $v^{2}$ if $C_{D}$ had remained constant. Hence, over this typically small range of values of $v, C_{D}$ often manifests a net decrease with increasing $V$ faster than $v^{2}$ increases, so $D=C_{D} \rho v^{2} / 2$ decreases with increasing $v$ (see Supplementary Note 11). Golf balls have dimples to facilitate laminar-to-turbulent flow transition at airspeeds low enough for golfers to achieve [23] [24] [25] [26]. Not only do the dimples decrease drag [23] [24] [25] [26], but they also increase lift [23] [24] [25] [26]. Thus they enhance golf balls' $C_{L} / C_{D}$ ratio and hence $L / D$ ratio not only via decreased $D$ but also via increased $L$ [23] [24] [25] [26]. Assists for laminar-to-turbulent transition are also employed, for example, in fluid-dynamic modeling and in small aircraft (see Supplementary Note 11). (c) As Mach 1 (the speed of sound) is approached from below, $C_{D}$ typically manifests a sharp peak, followed by a sharp dip at values of $v$ slightly above Mach 1 . The extra drag due to shock waves at and in the vicinity of Mach 1 is referred to as shock-wave drag (or sometimes simply as wave drag). (See Supplementary Note 12.)

It should be noted that, in the design of aircraft, even departures from the paramount $\rho v^{2}$ functional dependency of lift and/or drag smaller than those discussed in Items (a), (b), and (c) of the immediately preceding paragraph can yield modest but still significant improvements in aircraft energy efficiency [27] [28]: ${ }^{14}$ See the two immediately following paragraphs.

Not uncommonly, aerodynamic level flight at a given $V$ is more energy-efficient at lower $\rho$ and hence at higher $h_{A}$. This can obtain despite the required increase in angle of attack to above that which maximizes $C_{L} / C_{D}$ and hence $L / D$, consequently decreasing $C_{L} / C_{D}$ and hence $L / D$, as the penalty for increasing $C_{L}$ itself and hence $L$ itself sufficiently to maintain $L=m g$ in the face of decreased $\rho$ at fixed $v$ [recall Equations (9) and (10)]: up to a limit, $C_{L} / C_{D}$ and hence $L / D$ decreases more slowly with the required increase in

${ }^{14}$ See also the following articles: "Fuel economy in aircraft" (most recently revised in 2019) at https://www.wikipedia.org, and "AP4ATCO - Factors Affecting Aircraft Performance During Cruise" (most recently revised in 2013) at https://www.skybrary.aero/index.php/Main_Page. (The latter article is under construction at the time of this writing.) Not uncommonly, up to some limiting altitude, less power is required to maintain the same airspeed at higher altitudes than at lower ones: see Ref. [10], Sections XIV.4-XIV.5 (especially Figs. 297-300 and the associated discussions in Section XIV.5); also Ref. [13], pp. 49-53 and Chaps. VIII-IX. 
angle of attack than $\rho$ decreases. ${ }^{14}$ Of course this obtains only up to a limit: with continued increase in angle of attack $C_{L} / C_{D}$ and hence $L / D$ decreases ever more rapidly until stalling occurs. Higher-altitude aerodynamic level flight while maintaining the (smaller) angle of attack that maximizes $L / D$ would be at sufficiently faster $v$ to decrease the flight time $t$ more than it increases the required power $P_{A}$, and hence would increase energy efficiency $E_{A} / X=P_{A} t / X=P_{A} / v$ even more. But the engine(s) may not be capable of the required increase in $P_{A}$. Even if they are, in some cases increased $v$ may be detrimental [e.g., owing to encountering shock-wave drag if Mach 1 is approached too closely (see Supplementary Note 12), or to excessive aerodynamic heating]. A specific example of this (owing to encountering shock-wave drag if Mach 1 is approached too closely) is discussed in the immediately following paragraph.

As a specific example, the energy efficiency (distance $X$ per unit of fuel relative to the air, also relative to the ground if the wind is calm) of older commercial jet airliners was $\approx 43 \%$ higher at $35,000 \mathrm{ft}$ to $40,000 \mathrm{ft}(\approx 5 \mathrm{mi} / 100 \mathrm{lb}$ fuel $)$ than at $20,000 \mathrm{ft}$ ( $\approx 3.5 \mathrm{mi} / 100 \mathrm{lb}$ fuel) [27]. Assuming Curzon-Ahlborn [19] [20] engine efficiency ${ }^{7} 1-\left(T_{C} / T_{H}\right)^{1 / 2}$ and an atmosphere if not identical with then at least close to the U. S. Standard Atmosphere [29] [30] [31] [32] [33] (see Supplementary Note 13 ), only an $\approx 7 \%$ increase in energy efficiency can reasonably be attributed to lower atmospheric temperatures and hence to higher engine efficiency $\epsilon$ at $35,000 \mathrm{ft}$ to $40,000 \mathrm{ft}$ than at $20,000 \mathrm{ft}$ (see Section 2.4). [Assuming Carnot engine efficiency ${ }^{6} 1-\left(T_{C} / T_{H}\right)$ the figure is only $\approx 5 \%$, but the Curzon-Ahlborn [19] [20] engine efficiency ${ }^{7}$ is a more realistic estimate for real-world engines.] Thus improved aerodynamic efficiency rather than improved engine efficiency must have contributed a factor of $\approx 1.43 / 1.07 \approx 1.34$ to the improved energy efficiency at $35,000 \mathrm{ft}$ to $40,000 \mathrm{ft}$ over and above that at $20,000 \mathrm{ft}$. Perhaps improved $L / D$ ratios at given angles of attack at lower $\rho$ and hence at higher $h_{A}$ might contribute somewhat. But the major contribution to this improved energy efficiency-the only contribution if, as is usually at least approximately true, $L / D$ ratios at given angles of attack are independent of $\rho$ and hence of $h_{A}$-is the reduced power $P_{A}$ and hence reduced energy $E_{A}$ required to traverse a given distance $X$ at a given $v$ at lower $\rho$ and hence at higher $h_{A}$. This is not uncommon, despite the required increase in angle of attack to above that which maximizes $C_{L} / C_{D}$ and hence $L / D$, consequently decreasing $C_{L} / C_{D}$ and hence $L / D$, as the penalty for increasing $C_{L}$ itself and hence $L$ itself sufficiently to maintain $L=m g$ in the face of decreased $\rho$ at fixed $v$ [recall Equations (9) and (10)]: up to a limit, $C_{L} / C_{D}$ and hence $L / D$ decreases more slowly with the required increase in angle of attack than $\rho$ decreases. ${ }^{14}$ Of course this obtains only up to a limit: with continued increase in angle of attack $C_{L} / C_{D}$ and hence $L / D$ decreases ever more rapidly until stalling occurs. Higher-altitude aerodynamic level flight while maintaining the (smaller) angle of attack that would ordinarily maximize $L / D$ would ordinarily also be at sufficiently faster $v$ to decrease the flight time $t$ more than it increases the required power $P_{A}$, and hence would ordinarily increase energy efficiency 
$E_{A} / X=P_{A} t / X=P_{A} / v$ even more. But the engine(s) may not be capable of the required increase in $P_{A}$. Even if they are, in this case increased $v$ would be sufficiently close to Mach 1 to encounter shock-wave drag and consequently increased $D$ and thus decreased $L / D$ even at the optimum angle of attack, and hence also an increase in $P_{A}$ required to overcome the shock-wave drag over and above that owing to increased $v$. [See Item (c) of the first paragraph of this Section 3.1.2 and Supplementary Note 12.]

But, again, in this paper we focus mainly on the paramount $\rho v^{2}$ functional dependency of both lift and drag, which is the first-order dependency upon $\rho$ and upon $v$. Considerations of departures from the first-order $\rho v^{2}$ functional dependency [27] [28], whether the large departures as per Items (a), (b), and (c) discussed in the first paragraph of this Section 3.1.2, or the smaller departures that can yield modest but still significant improvements in aircraft energy efficiency [27] [28] discussed in the second, third, and fourth paragraphs thereof (see also Supplementary Notes 5, 6, 8-12, and 14), require mathematically complex and detailed fully-quantitative analyses based on rigorous application of fluid dynamics, e.g., computational fluid dynamics. Such analyses are of course essential in the actual design of aircraft [2]-[15] [27] [28], ${ }^{14}$ but we do not attempt them in this paper: our analyses are qualitative to semiquantitative. Thus we conceal the difficult and complex physics underlying departures from the first-order $\rho v^{2}$ functional dependency [27] [28] within $C_{D}$ and $C_{L}$, specifically, within departures of $C_{D}$ and $C_{L}$ from constancy. Despite the three major exceptions (a), (b), and (c) discussed in the first paragraph of this Section 3.1.2, and also despite ones that are more minor (but still essential in the actual design of aircraft [2]-[15] [27] [28] $)^{14}$ such as discussed in the second, third, and fourth paragraphs thereof (see also Supplementary Notes 5, 6, 8-12, and 14), the $\rho v^{2}$ functional dependency of both drag and lift is the paramount, first-order, functional dependency [2]-[15] [27] [28].

\subsection{The Low-Density/High-Altitude Limit of Aerodynamic Level Flight in Earth's Atmosphere}

If $\rho$ is so small that even the minimum airspeed required for aerodynamic level flight equals or exceeds the speed

$v_{\text {orbit,Earth }}=\left(G M_{\text {Earth }} / r\right)^{1 / 2} \doteq\left(G M_{\text {Earth }} / R_{\text {Earth }}\right)^{1 / 2} \approx 8 \times 10^{3} \mathrm{~m} / \mathrm{s}$ required for minimum-altitude circular-orbit ballistic spaceflight about Earth, ${ }^{4}$ then all flight about Earth must be ballistic rather than aerodynamic. We now estimate how small $\rho$ must be and how high the altitude in Earth's atmosphere must be for this to obtain. As discussed in Section 3.1, aerodynamic forces of lift and drag are typically, at least approximately, proportional to $\rho v^{2}$ [2]-[15]. Thus if $v_{\min }\left(\rho_{0 \text {,Earth }}\right)$ is the minimum airspeed at which an aircraft can maintain $L=m g$ and hence aerodynamic level flight at sea-level air density $\rho_{0 \text {,Earth }} \approx 1 \mathrm{~kg} / \mathrm{m}^{3}$ (maximizing $L$ may require flight at less-than-maximum $L / D$ : see Section 3.1.2 and Supplementary Notes 5, 6, and 9), then even neglecting all 
practical considerations - the most obvious and most general of practical considerations being power requirements and frictional aerodynamic heating-the absolute minimum air density $\rho_{\text {min,abs,Earth }}$ at which it can maintain $L=m g$ and hence aerodynamic level flight at

$$
\begin{aligned}
v<v_{\text {min }}\left(\rho_{\text {min,abs,Earth }}\right) & \approx v_{\text {orbit,Earth }} \approx 8 \times 10^{3} \mathrm{~m} / \mathrm{s} \text { is }{ }^{15} \\
\rho_{\text {min,abs,Earth }} & \approx \rho_{0, \text { Earth }}\left[\frac{v_{\text {min }}\left(\rho_{0, \text { Earth }}\right)}{v_{\text {orbit,Earth }}}\right]^{2} \approx \rho_{0 \text {,Earth }}\left[\frac{v_{\text {min }}\left(\rho_{0, \text { Earth }}\right)}{8 \times 10^{3} \mathrm{~m} / \mathrm{s}}\right]^{2} .
\end{aligned}
$$

The minimum airspeeds $v_{\min }\left(\rho_{0 \text {,Earth }}\right)$ required for aerodynamic level flight at sea-level air density $\rho_{0 \text {,Earth }} \approx 1 \mathrm{~kg} / \mathrm{m}^{3}$ of any aircraft obtain for the lightest unmanned model airplanes, which are limited to indoor flights, and to outdoor flights only if the wind is calm. (For hovering flight these minimum airspeeds are the root-mean-square average, taken over the geometrical area of the airfoils, of airspeeds of the airfoils, be they blades of a helicopter, or wings of a hovering hummingbird or insect.) These minimum airspeeds $v_{\text {min }}\left(\rho_{0 \text {,Earth }}\right)$ at sea-level air density $\rho_{0 \text {,Earth }} \approx 1 \mathrm{~kg} / \mathrm{m}^{3}$ are somewhat less than $1 \mathrm{~m} / \mathrm{s}$, or $\approx 10^{-4}$ of

${ }^{15}$ At ground speed $v$, in level flight a fraction $\left(v / v_{\text {orbit, } x}\right)^{2}$ of an aircraft's weight $m g_{x}$ is offset by centrifugal force about the center of a gravitating body (in particular about Earth's center or Mars' center). (In this Footnote 15, the subscript $\mathrm{x}$ can refer to any gravitating body with an atmosphere wherein aerodynamic flight is possible, but we focus on Earth and Mars.) Thus, as per pp. 29-30 of Ref. [45], the boundary between aerodynamic flight and spaceflight can be construed at half of an aircraft's weight $m g_{\mathrm{x}}$ being offset by centrifugal force. This obtains at ground speed $v_{\text {obiti, } x} / 2^{1 / 2}$. Since $v_{\text {orbiti, } x}$, or even $v_{\text {orbiti, } x} / 2^{1 / 2}$, is much faster than any winds on either Earth or Mars, in this Footnote 15 we can neglect any differences between ground speed and airspeed and refer simply to speed $v$. Bear in mind the paramount $\rho v^{2}$ functional dependency of aerodynamic lift as per Section 3.1 and hence that variation of $C_{L}$ (at any given angle of attack) with $\rho$ and with $V$, if any, is typically small. To maintain level flight with half of an aircraft's weight $\frac{1}{2} m g_{\mathrm{x}}$ offset by centrifugal force as per pp. 29-30 of Ref. [45], if the other half is to be offset by aerodynamic lift given $\rho=\rho_{\text {min,abs,half, } x}$ flight speed must be $v_{\text {orbit, } x} / 2^{1 / 2}$ as per Equations (9) and (10):

$$
\begin{aligned}
\frac{1}{2} L & =\frac{1}{2} m g_{x}=\frac{1}{4} C_{L} A_{\text {wing, geom }} \rho_{\text {min, abs, half }, x}\left(v_{\text {orbit, } x} / 2^{1 / 2}\right)^{2} \\
& =\frac{1}{8} C_{L} A_{\text {wing,geom }} \rho_{\text {min, abs, half }, x} v_{\text {orbit }, x}^{2}
\end{aligned}
$$

(employing notation as per Section 3.2 and the last

three paragraphs of Section 3.3.1). Neglecting the contribution of centrifugal force about Earth's center or Mars' center as per Sections 3.2 and 3.3.1, and hence requiring all of an aircraft's weight $m g_{\mathrm{x}}$ to be offset by aerodynamic lift given $\rho=\rho_{\text {min,abs,all, }}$, by Equations (9) and (10) flight speed must be $v_{\text {orbit, } x}$ :

$L=m g_{\mathrm{x}}=\frac{1}{2} C_{L} A_{\text {wing, geom }} \rho_{\text {min, abs,all, },} v_{\text {orbiti, } x}^{2}$.

Thus within the approximation that $\rho v^{2}$ is a conserved quantity, independent of $\rho$ (and hence of flight altitude on either Earth or Mars) and of $v, \rho_{\text {min,abs,alf }, x}=4 \rho_{\text {min,abs,all, }}$ : with the help of centrifugal force the absolute lower limit of air density $\rho_{\text {min,abs,x }}$ for aerodynamic level flight is 4 times that without its help. Thus within this approximation as per the last six paragraphs of Section 3.1.1 the power required for aerodynamic level flight at the absolute lower limit of air density $\rho_{\text {min,abs, } x}$, and hence also (since this power must be frictionally dissipated) the consequent frictional aerodynamic heating, is less by a factor of $(1 / 4)^{1 / 2}=1 / 2$ with the help of centrifugal force than without its help. But, at any rate, in Section 3.2 and the last three paragraphs of Section 3.3.1, we give only in-the-ballpark estimates. 
$v_{\text {orbit,Earth }} \approx 8 \times 10^{3} \mathrm{~m} / \mathrm{s}$ required for minimum-altitude circular-orbit ballistic spaceflight about Earth, ${ }^{4}$ say, $v_{\min }\left(\rho_{0, \text { Earth }}\right) \approx 0.8 \mathrm{~m} / \mathrm{s}$. [By Equations $(9)$ and (10), if $C_{L} \approx 1$, taking $\rho=\rho_{0 \text {,Earth }} \approx 1 \mathrm{~kg} / \mathrm{m}^{3}$ and $g=9.8 \mathrm{~m} / \mathrm{s}^{2}$, $v \approx 10^{-4} v_{\text {orbitEarth }} \approx 0.8 \mathrm{~m} / \mathrm{s}$ corresponds to a wing loading of $\mathrm{mg} / A_{\text {wing,geom }} \approx \frac{1}{2} \rho_{0, \text { Earth }} v^{2} \approx\left(\frac{1}{2} \times 1 \times 0.8^{2}\right) \mathrm{N} / \mathrm{m}^{2} \approx 0.3 \mathrm{~N} / \mathrm{m}^{2}$ or $m / A_{\text {wing,geom }} \approx 0.03 \mathrm{~kg} / \mathrm{m}^{2}$. Thus, putting $v_{\text {min }}\left(\rho_{0, \text { Earth }}\right) \approx 0.8 \mathrm{~m} / \mathrm{s}$ in Equation (15), if $\rho \lesssim \rho_{\text {min,abs,Earth }} \approx\left(10^{-4}\right)^{2} \rho_{0 \text {,Earth }}=10^{-8} \rho_{0 \text {,Earth }} \approx 10^{-8} \mathrm{~kg} / \mathrm{m}^{3}$, corresponding to altitudes $h \gtrsim h_{A \text {, max,abs,Earth }} \approx 130 \mathrm{~km} \approx 430000 \mathrm{ft}$ above sea level in Earth's atmosphere [29]-[34] (see also Supplementary Note 13), then the minimum airspeeds required for aerodynamic level flight of even these lightest unmanned model airplanes equal or exceed $v_{\text {orbit,Earth }} \approx 8 \times 10^{3} \mathrm{~m} / \mathrm{s}$ required for minimum-altitude circular-orbit ballistic spaceflight about Earth..$^{15}$ Hence all flight about Earth must then be ballistic rather than aerodynamic. ${ }^{15}$

This estimate of the low-density/high-altitude limit for aerodynamic level flight about Earth is an ultimate limit that neglects all practical difficulties. To re-emphasize, the most obvious and most general of practical difficulties are the required power $P_{A}$ and especially the consequent frictional aerodynamic heating, the latter being equal to $P_{A}$ because $P_{A}$ is ultimately thermally dissipated via frictional aerodynamic heating. Other practical difficulties, which we do not consider, include the reduction of the power available from air-breathing engines with decreasing $\rho$ and hence with increasing $h_{A}$ (obviously aerodynamic level flight is possible if and only if the maximum available power exceeds, or at the very least equals, the required power $\left.P_{A}\right),{ }_{,}^{13}$ and practical difficulties that are specific for given types of aircraft, e.g., maximum airspeeds for propeller airplanes, ${ }^{16}$ and minimum and maximum airspeeds for various types of jets. ${ }^{17}$ By the last five paragraphs of Section 3.1.1, $P_{A}$, and hence also the consequent frictional aerodynamic heating and the required rate of heat dissipation, is, at least approximately, proportional to $\rho^{-1 / 2}$ [2]-[15]. Thus the practical low-density/high-altitude limit of aerodynamic level flight is considerably more conservative than the ultimate limit. The altitude record for aerodynamic level flight in Earth's atmosphere as of this writing, $\approx 124000 \mathrm{ft} \approx 38 \mathrm{~km}$, corresponds to $\rho$ slightly smaller than $10^{-2} \rho_{0, \text { Earth }} \approx 10^{-2} \mathrm{~kg} / \mathrm{m}^{3} .{ }^{18}$ Perhaps a reasonable estimate of the practical low-density/high-altitude limit of aerodynamic level flight in Earth's atmosphere, even if attainable only by the lightest

\footnotetext{
${ }^{16}$ See Ref. [14], pp. 81-82.

${ }^{17}$ See Ref. [9], pp. 176-177; Ref. [14], pp. 82-86 and 146-150; the following articles at https://www.skybrary.aero/index.php/Main_Page: "Jet Engine" (most recently revised in 2017), "Turboprop Engine" (most recently revised in 2017), "Turbojet Engine" (most recently revised in 2017), "Turbofan Engine" (most recently revised in 2016), "Geared Turbofan Engine" (most recently revised in 2017), "Ramjet" (most recently revised in 2017), and "Scramjet" (most recently revised in 2017); and the following articles (all most recently revised in 2019) at https://www.wikipedia.org: "Jet engine", “Jet engine performance", “Turboprop”, “Turbojet”, “Turbofan”, “Ramjet”, “Scramjet”, and "Shcramjet".

${ }^{18}$ See also the following article (most recently revised in 2019) at https://www.wikipedia.org: "Flight altitude record".
} 
unmanned model airplanes, is $\rho_{\text {min,prac,Earth }} \approx 10^{-3} \rho_{0 \text {,Earth }} \approx 10^{-3} \mathrm{~kg} / \mathrm{m}^{3}$, corresponding to an altitude of $h_{A \text {, max,prac,Earth }} \approx 180000 \mathrm{ft} \approx 55 \mathrm{~km}$. (This is also approximately the practical low-density/high-altitude limit of balloons in Earth's atmosphere as of this writing. ${ }^{18}$ )

Note that, by Equation (2) and the last six paragraphs of Section 3.1.1 (neglecting exceptions to the paramount $\rho v^{2}$ functional dependency as per Section 3.1.2), the energy $E_{A}$ required for aerodynamic level flight of an aircraft of weight $m g$ traversing given distance $X$ is not greater at the practical-or even the ultimate-low-density/high-altitude limit of aerodynamic level flight than at sea level. Difficulties arise only because $E_{A}$ must be expended faster and hence thermally dissipated faster (frictional aerodynamic heating!) in thinner air$P_{A}=\partial E_{A} / \partial t \propto \rho^{-1 / 2}$.

\subsection{Level Flight in Rarefied and Dense Aerodynamic Media: Mars Airplanes and Underwater Airplanes}

\subsubsection{Mars Airplanes}

Atmospheric density at low altitudes on Mars, $\rho_{0, \text { Mars }} \approx(1 / 70) \mathrm{kg} / \mathrm{m}^{3}$, is $\approx 1 / 70$ of $\rho_{0, \text { Earth }} \approx 1 \mathrm{~kg} / \mathrm{m}^{3}$ as obtains on Earth [35] [36] [37] (see also Supplementary Note 14). But this density is nevertheless substantial enough for Mars airplanes to be within the practical, not merely ultimate, limit of aerodynamic level flight, especially given that Mars' surface gravity is only $\approx 0.38$ that of Earth. By the last six paragraphs of Section 3.1.1, if all other things except $\rho$ were equal (e.g., same airplane, same angle of attack, etc.), then a Mars low-altitude airplane must fly $\approx(1 / 70)^{-1 / 2}=70^{1 / 2} \approx 8.4$ times faster than an identical Earth low-altitude airplane and requires $\approx(1 / 70)^{-1 / 2}=70^{1 / 2} \approx 8.4$ times as much power to sustain aerodynamic level flight. Even this is within the practical, not merely ultimate, limit of aerodynamic level flight. But we must still consider one important factor that is unequal in favor of Mars-Mars' weaker gravity: $g$ on Mars is only $\approx 0.38$ of $g$ on Earth [35] [36] [37]. Thus by the first line of Equation (14), all other things except $\rho$ and $g$ being equal (e.g., same airplane, same angle of attack, etc.), to maintain aerodynamic level flight airspeed $v \propto(\mathrm{mg} / \rho)^{1 / 2}$ is required. Hence a Mars low-altitude airplane need fly only $\approx[0.38 \div(1 / 70)]^{1 / 2}=(0.38 \times 70)^{1 / 2} \approx 5.2$ times faster than an identical Earth low-altitude airplane and requires only $\approx 0.38^{3 / 2} \div(1 / 70)^{1 / 2}=0.38^{3 / 2} \times 70^{1 / 2} \approx 2.0$ times as much power to sustain aerodynamic level flight. This is certainly not merely within, but well within, the practical, not merely ultimate, limit of aerodynamic level flight. Moreover, by Equation (2), the last six paragraphs of Section 3.1.1, and the last paragraph of Section 3.2, all other things except $g$ being equal (e.g., same airplane, same angle of attack, etc.), a Mars airplane of mass $m$ requires only $\approx 0.38$ as much energy $E_{A}$ as an identical Earth airplane of the same mass $m$ to traverse a given horizontal distance $X$. Thus with respect to $E_{A}$ aerodynamic level flight is easier by a factor of $\approx 0.38$ on Mars than on Earth. Note that this greater ease by a factor of $\approx 0.38$ with respect to $E_{A}$ of aerodynamic level flight on Mars than on Earth is due solely to $g$ on Mars being $\approx 0.38$ 
of $g$ on Earth [35] [36] [37]: it obtains at all atmospheric densities and hence at all aerodynamic flight altitudes. Corresponding to given $\rho$, say, comparing high-altitude aerodynamic level flight on Earth with otherwise-identical low-altitude aerodynamic level flight on Mars, by Equation (14) a Mars airplane need fly only $\approx 0.38^{1 / 2} \approx 0.62$ as fast as an identical Earth airplane and requires only $\approx 0.38^{3 / 2} \approx 0.23$ as much power to sustain aerodynamic level flight.

Recalling the third and fourth paragraphs of Section 3.1.2, we also note that aerodynamic level flight at a given $v$ can be more energy-efficient at lower $\rho$ as on Mars. This can obtain despite the required increase in angle of attack to above that which maximizes $C_{L} / C_{D}$ and hence $L / D$, consequently decreasing $C_{L} / C_{D}$ and hence $L / D$, as the penalty for increasing $C_{L}$ itself and hence $L$ itself sufficiently to maintain $L=m g$ in the face of decreased $\rho$ [recall Equations (9) and (10)]: up to a limit, $C_{L} / C_{D}$ and hence $L / D$ decreases more slowly with the required increase in angle of attack than $\rho$ decreases. ${ }^{14}$ Of course this obtains only up to a limit: with continued increase in angle of attack $C_{L} / C_{D}$ and hence $L / D$ decreases ever more rapidly until stalling occurs. Aerodynamic level flight at lower $\rho$ as on Mars while maintaining the (smaller) angle of attack that maximizes $L / D$ would be at sufficiently faster $v$ to decrease the flight time $t$ more than it increases the required power $P_{A}$, and hence would increase energy efficiency $E_{A} / X=P_{A} t / X=P_{A} / v$ even more. But the engine(s) may not be capable of the required increase in $P_{A}$. Even if they are, in some cases increased $V$ may be detrimental (recall the third and fourth paragraphs of Section 3.1.2).

What is more, owing to Mars' weaker gravity, atmospheric density decreases more slowly with increasing altitude on Mars than on Earth, even in the face of the higher molecular weight and lower temperature of Mars' atmosphere. The scale height [29]-[37] in Earth's atmosphere, corresponding to an $e$-fold decrease in atmospheric density, is $\approx 8.5 \mathrm{~km}$. In Mars' atmosphere it is $\approx 11 \mathrm{~km}$. Since, at low altitudes, density in Mars' atmosphere is $\approx 1 / 70$ of that in Earth's atmosphere [35] [36] [37] (see also Supplementary Note 14), the ratio $\mathcal{R}$ of density in Mars' atmosphere to that in Earth's atmosphere at altitude $h$ is

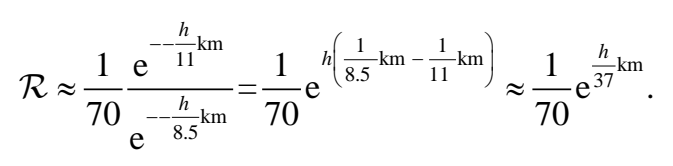

At $h \approx 157 \mathrm{~km}, \mathcal{R} \approx 1$, i.e., density is approximately equal in Mars' and Earth's atmospheres. At all higher altitudes, Mars' atmosphere is denser than Earth's, and in increasing ratio $\mathcal{R}$ with increasing altitude $h$.

Thus the practical low-density limit of aerodynamic level flight in Earth's atmosphere $\rho_{\text {min,prac,Earth }} \approx 10^{-3} \rho_{0 \text {,Earth }} \approx 10^{-3} \mathrm{~kg} / \mathrm{m}^{3}$ corresponds (solving $10^{-3} \approx \frac{1}{70} \mathrm{e}^{-h / 1 \mathrm{~km}}$ ) to an altitude of $h \approx 29 \mathrm{~km} \approx 95000 \mathrm{ft}$ in Mars' atmosphere. This is also approximately the practical low-density/high-altitude limit of balloons in Mars' atmosphere. The practical low-density limit of buoyant flight 
$\rho \approx 10^{-3} \mathrm{~kg} / \mathrm{m}^{3}$ is equal in Earth's and Mars' atmospheres because lower $g$ on Mars reduces both weight and buoyancy by the same factor of $\approx 0.38$. Indeed for this reason it is $\rho \approx 10^{-3} \mathrm{~kg} / \mathrm{m}^{3}$ in any atmosphere of any planet. This is strictly true for buoyant flight via vacuum airships, which obtain buoyant lift equal to atmospheric density $\rho$ per unit volume of (essentially) perfect vacuum, or $(1-\mathcal{F}) \rho$ per unit volume of imperfect or partial vacuum (e.g., hot-air balloons) of density $\mathcal{F} \rho$ (of course $0<\mathcal{F}<1$ ). Depressurized (as opposed to hot-air) vacuum airships are more practicable on Mars than on Earth: owing to the higher molecular weight and lower temperature of Mars' atmosphere, its ratio of pressure to density is smaller than that of Earth's atmosphere. See: Clarke, J.-P.; Rimoli, J.; Gloyd, J. T.; Logarzo, H.; Kraus, J. (2018) Evacuated Airship for Mars Missions (Georgia Tech Air Transportation Laboratory) at https://ntrs.nasa.gov/search.jsp?R=20180006789 (left-click on "View Document") and Vacuum airship (most recently revised in 2019) at

https://www.wikipedia.org. For buoyant flight via lifting gas of density $\mathcal{F} \rho$ (of course $0<\mathcal{F}<1$ ) buoyant lift equals $(1-\mathcal{F}) \rho$ per unit volume of lifting gas. Since for a lifting gas $\mathcal{F}$ equals the ratio of the molecular weight of the lifting gas to that of the ambient atmosphere, unlike for vacuum airships (or partial-vacuum airships such as hot-air balloons) $\mathcal{F}$ is larger for a given lifting gas in a higher-molecular-weight atmosphere such as Mars' atmosphere than in a lower-molecular-weight one such as Earth's. (Obviously, $0<\mathcal{F} \ll 1$ is required if the practical low-density limit of buoyant flight $\rho \approx 10^{-3} \mathrm{~kg} / \mathrm{m}^{3}$ is to be attained.)

But the practical high-altitude limit $h_{A \text {, max, prac,Mars }}$ of aerodynamic level flight on Mars is much higher than this, because we still must consider one important thing that is unequal in favor of Mars-Mars' weaker gravity: $g$ on Mars is $\approx 0.38$ of $g$ on Earth. By Equation (14) the power $P_{A \text {,Mars }}$ required for aerodynamic level flight in Mars' atmosphere at the altitude where

$$
\begin{aligned}
& 0.38^{3 / 2} / \rho_{\min , \text { prac,Mars }}^{1 / 2}=1^{3 / 2} / \rho_{\min , \text { prac,Earth }}^{1 / 2}=1 / \rho_{\min , \text { prac,Earth }}^{1 / 2} \approx 1 /\left(10^{-3} \mathrm{~kg} / \mathrm{m}^{3}\right)^{1 / 2}, \text { i.e., } \\
& \text { where } \rho_{\text {min, prac,Mars }}=0.38^{3} \rho_{\text {min,prac,Earth }} \approx 0.38^{3} \times 10^{-3} \mathrm{~kg} / \mathrm{m}^{3} \approx 5 \times 10^{-5} \mathrm{~kg} / \mathrm{m}^{3},
\end{aligned}
$$

equals the power $P_{A \text {,Earth }}$ required for aerodynamic level flight in Earth's atmosphere at the altitude $h_{A \text {, max, prac,Earth }} \approx 180000 \mathrm{ft} \approx 55 \mathrm{~km}$ where $\rho \approx \rho_{\text {min,prac,Earth }} \approx 10^{-3} \mathrm{~kg} / \mathrm{m}^{3}$, our estimate of the practical

low-density/high-altitude limit of aerodynamic level flight in Earth's atmosphere (recall the third paragraph of Section 3.2). Thus probably a reasonable estimate of the practical low-density/high-altitude limit of aerodynamic level flight in Mars' atmosphere, even if attainable only by the lightest unmanned model airplanes (recall Section 3.2), is $\rho_{\text {min,prac,Mars }} \approx 5 \times 10^{-5} \mathrm{~kg} / \mathrm{m}^{3}$, which corresponds (solving $5 \times 10^{-5} \approx \frac{1}{70} \mathrm{e}^{-h_{A, \text { max }, \text { prac, Mars }} / 1 \mathrm{~km}}$ ) to an altitude of

$h_{A \text {, max, prac,Mars }} \approx 62 \mathrm{~km} \approx 204000 \mathrm{ft}$ in Mars' atmosphere-slightly higher than $h_{\text {A,max, prac,Earth }} \approx 180000 \mathrm{ft} \approx 55 \mathrm{~km}$. Low $g$ on Mars, $\approx 0.38$ of $g$ on Earth, implies a 
reasonable estimate of $\rho_{\text {min,prac,Mars }}$ being lower than $\rho_{\text {min,prac,Earth }}$. This lower required density, combined with $g$ on Mars $\approx 0.38$ of $g$ on Earth rendering the scale height [29]-[37] being $\approx 11 / 8.5 \approx 1.3$ times greater in Mars' atmosphere than in Earth's atmosphere even in the face of the higher molecular weight and lower temperature of Mars' atmosphere, contributes to $h_{A \text {, max,prac,Mars }}$ being slightly higher than $h_{A \text {,max,prac,Earth }}$ despite Martian low-altitude air density being only $\approx 1 / 70$ of that on Earth [35] [36] [37] (see also Supplementary Note 14). This practical low-density/high-altitude limit considers $P_{A}$ and consequent frictional aerodynamic heating: $P_{A}$ must be thermally dissipated. (Note that while the practical high-altitude limits of aerodynamic level flight and of buoyant flight are approximately equal in Earth's atmosphere, ${ }^{18}$ the former is considerably higher than the latter in Mars' atmosphere. As noted in the immediately preceding paragraph, the practical high-altitude limit of buoyant flight corresponds to $\rho \approx 10^{-3} \mathrm{~kg} / \mathrm{m}^{3}$ on Mars as on Earth because lower $g$ on Mars reduces both weight and buoyancy by the same factor of $\approx 0.38$-indeed for this reason it is $\rho \approx 10^{-3} \mathrm{~kg} / \mathrm{m}^{3}$ in any atmosphere of any planet.)

Now let us consider the ultimate, as opposed to the practical, low-density/high-altitude limit of aerodynamic level flight in Mars' atmosphere-corresponding to the speed of aerodynamic level flight of the lightest unmanned model airplanes equaling minimum-altitude circular-orbital speed on Mars, neglecting all practical considerations, the most obvious and most general of which are power requirements and frictional aerodynamic heating. Consider indoor flight of the lightest unmanned model airplanes on Mars with indoor air density maintained equal to that at sea level on Earth, i.e., $\rho_{0 \text {,Earth }} \approx 1 \mathrm{~kg} / \mathrm{m}^{3}$. Since $g$ on Mars is $\approx 0.38$ of $g$ on Earth, by the first line of Equation (14) the speed required to maintain indoor aerodynamic level flight of the lightest unmanned model airplanes on Mars at indoor air density $\rho_{0, \text { Earth }} \approx 1 \mathrm{~kg} / \mathrm{m}^{3}$ is $v_{\text {min,Mars }}\left(\rho_{0, \text { Earth }}\right) \approx 0.38^{1 / 2} \approx 0.62$ of the $\approx 0.8 \mathrm{~m} / \mathrm{s}$ required on Earth, i.e., $\approx 0.5 \mathrm{~m} / \mathrm{s}$. The speed

$v_{\text {orbit,Mars }}=\left(G M_{\text {Mars }} / r\right)^{1 / 2} \approx\left(G M_{\text {Mars }} / R_{\text {Mars }}\right)^{1 / 2} \approx 3.6 \mathrm{~km} / \mathrm{s}$ required for minimum-altitude circular-orbit ballistic spaceflight on Mars is $\approx 0.45$ times $v_{\text {orbit,Earth }} \approx 8 \times 10^{3} \mathrm{~m} / \mathrm{s} .{ }^{4}$ Thus for Mars' atmosphere, applying and slightly modifying Equation (15) yields ${ }^{15}$

$$
\begin{aligned}
\rho_{\text {min,abs,Mars }} & \approx \rho_{0, \text { Earth }}\left[\frac{v_{\text {min,Mars }}\left(\rho_{0, \text { Earth }}\right)}{v_{\text {orbit,Mars }}}\right]^{2} \approx \rho_{0, \text { Earth }}\left[\frac{0.62 v_{\text {min,Earth }}\left(\rho_{0, \text { Earth }}\right)}{0.45 v_{\text {orbit,Earth }}}\right]^{2} \\
& \approx \rho_{0, \text { Earth }}\left[\frac{0.62(0.8 \mathrm{~m} / \mathrm{s})}{0.45\left(8 \times 10^{3} \mathrm{~m} / \mathrm{s}\right)}\right]^{2} \approx \rho_{0, \text { Earth }}\left(\frac{0.62}{0.45} \times 10^{-4}\right)^{2} \\
& \approx 1.9 \times 10^{-8} \rho_{0, \text { Earth }} \approx 1.9 \times 10^{-8} \mathrm{~kg} / \mathrm{m}^{3} \\
& \approx 1.9 \rho_{\text {min,abs,Earth }} \approx 1.3 \times 10^{-6} \rho_{0, \text { Mars }} .
\end{aligned}
$$

Solving $1.3 \times 10^{-6} \approx \mathrm{e}^{-h_{A, \text { max,abs,Mars }} / 11 \mathrm{~km}}$ yields $h_{A, \text { max,abs,Mars }} \approx 149 \mathrm{~km} \approx 490000 \mathrm{ft}$ - slightly higher than $h_{A, \text { max,abs,Earth }} \approx 130 \mathrm{~km} \approx 430000 \mathrm{ft}$. The two reasons for 
this high value $h_{A \text {,max,abs,Mars }} \approx 149 \mathrm{~km} \approx 490000 \mathrm{ft}$ on Mars are $g$ on Mars being $\approx 0.38$ of $g$ on Earth and Mars' atmospheric scale height [29]-[37] $(\approx 11 \mathrm{~km})$ being $\approx 11 / 8.5 \approx 1.3$ times larger than Earth's $(\approx 8.5 \mathrm{~km})$. Indeed only owing to the first reason does the second reason obtain even in the face of the higher molecular weight and lower temperature of Mars' atmosphere [35] [36] [37] (see also Supplementary Note 14).

It is perhaps worthwhile to re-emphasize that, by Equation (2), the last six paragraphs of Section 3.1.1, the last paragraph of Section 3.2, and the first paragraph of this Section 3.3.1 (neglecting exceptions to the paramount $\rho v^{2}$ functional dependency as per Section 3.1.2), the energy $E_{A, \text { Mars }}$ required for aerodynamic level flight of an aircraft of mass $m$ traversing given distance $X$ is not greater at the practical—or even ultimate-low-density/high-altitude limit of aerodynamic level flight on Mars than at low altitudes on Mars: $E_{A, \text { Mars }} \approx 0.38$ of $E_{A \text {,Earth }}$ required to traverse $X$ at sea level or any higher altitude on Earth. Difficulties arise onlybecause $E_{A}$ must be expended faster and hence thermally dissipated faster (frictional aerodynamic heating!) in thinner air on Mars as on Earth as anywhere- $P_{A}=\partial E_{A} / \partial t \propto \rho^{-1 / 2}$.

\subsubsection{Underwater Airplanes on Earth and (Hypothetically) on Mars}

Now let us consider, even if only hypothetically as a thought experiment, a fully-submerged underwater airplane on Earth, even though no such craft actually exists and one is extremely unlikely to ever be built. (An underwater airplane, which cruises fully submerged, should not be confused with a hydrofoil, which typically except for its wings cruises above water.) Let our underwater airplane be of density $(\mathfrak{N}+1) \times 10^{3} \mathrm{~kg} / \mathrm{m}^{3}$, the volume of all of its solid parts be $V \mathrm{~m}^{3}$, and hence its mass be $m=(\mathfrak{N}+1) \times 10^{3} V \mathrm{~kg}$. Let us compare our underwater airplane to a standard (atmospheric) airplane on Earth, of identical size and shape, but of density $\mathfrak{N} \times 10^{3} \mathrm{~kg} / \mathrm{m}^{3}$ and hence of mass $m=\mathfrak{N} \times 10^{3} \mathrm{~V} \mathrm{~kg}$. Our underwater airplane's extra $10^{3} \mathrm{~kg} / \mathrm{m}^{3}$ of density offsets the buoyancy provided by water ( $\rho_{\text {water }} \approx 10^{3} \mathrm{~kg} / \mathrm{m}^{3}$ ), so that its underwater flight can be evenhandedly compared with atmospheric flight of our standard (atmospheric) airplane. Thus our underwater airplane's extra $10^{3} \mathrm{~kg} / \mathrm{m}^{3}$ of density renders water its aerodynamic medium as opposed to its buoyant medium, consistently with air being our standard (atmospheric) airplane's aerodynamic medium. This facilitates an evenhanded comparison between our standard (atmospheric) airplane's aerodynamic level flight in air and our underwater airplane's aerodynamic level flight in water. We take $\mathfrak{N}$ sufficiently larger than the density of air at sea level, $\rho_{0 \text {,Earth }} \approx 1 \mathrm{~kg} / \mathrm{m}^{3}$, that the buoyancy provided by air for our standard (atmospheric) airplane can be neglected (this easily obtains, because all solids are hundreds to thousands of times as dense as air at sea level), and we assume high enough Reynolds numbers for both airplanes that skin-friction drag is small compared to pressure drag for both airplanes (see Supplementary Note 10). Water is $\approx 800$ times as dense as air at sea level. Thus, by the last six paragraphs of 
Section 3.1.1, our underwater airplane need fly only $\approx 800^{-1 / 2} \approx 1 / 28$ as fast as our standard (atmospheric) airplane at or near sea level to sustain level flight, and by Equation (14) requires only $\approx 800^{-1 / 2} \approx 1 / 28$ as much power to sustain level flight as our standard (atmospheric) airplane at or near sea level. But our underwater airplane takes $\approx 800^{1 / 2} \approx 28$ times as long to traverse a given horizontal distance $X$ as our standard (atmospheric) airplane. Hence by Equation (2) and the last six paragraphs of Section 3.1.1, our Earth underwater airplane requires the same energy $E_{A}$ as our standard (atmospheric) Earth airplane to traverse a given horizontal distance $X$.

Recalling the last six paragraphs of Section 3.1.1 and that $g$ on Mars is $\approx 0.38$ of $g$ on Earth, if atmospheric pressure on Mars was high enough for liquid water to exist, then both the required flight speed and the required power for aerodynamic flight of a Mars underwater airplane would by Equation (14) be $\approx 0.38^{1 / 2} \approx 0.62$ of the values required for an identical Earth underwater airplane, both underwater airplanes being of density $(\mathfrak{N}+1) \times 10^{3} \mathrm{~kg} / \mathrm{m}^{3}$ and mass $m=(\mathfrak{N}+1) \times 10^{3} \mathrm{~V} \mathrm{~kg}$. (Atmospheric pressure on Mars was high enough for liquid water to exist in the past [37] but perhaps by a smaller margin than generally believed [38]. ${ }^{19}$ ) Also, by Equation (2) and the last six paragraphs of Section 3.1.1, a Mars underwater airplane requires the same energy $E_{A, \text { Mars }}$ to traverse given horizontal distance $X$ as a standard (atmospheric) Mars airplane identical except for being of density $\mathfrak{N} \times 10^{3} \mathrm{~kg} / \mathrm{m}^{3}$ and hence of mass $m=\mathfrak{N} \times 10^{3} V \mathrm{~kg}$. By Equation (2) and comparison with Section 3.3.1, this energy $E_{A, \text { Mars }}$ is $\approx 0.38$ times $E_{A \text {,Earth }}$ required to traverse the same horizontal distance $X$ by an identical Earth underwater airplane of density

$(\mathfrak{N}+1) \times 10^{3} \mathrm{~kg} / \mathrm{m}^{3}$ and mass $m=(\mathfrak{N}+1) \times 10^{3} V \mathrm{~kg}$, or by a standard (atmospheric) Earth airplane identical except for being of density $\mathfrak{N} \times 10^{3} \mathrm{~kg} / \mathrm{m}^{3}$ and hence of mass $m=\mathfrak{N} \times 10^{3} \mathrm{~V} \mathrm{~kg}$.

\subsection{Dual-Density Flight: Hydrofoils}

Hydrofoils should be classified as aircraft because their lift obtains primarily if not essentially entirely aerodynamically rather than via buoyancy, even though the density $\rho$ of their aerodynamic medium (water) is $\approx 800$ times that of air at sea level. By lifting the hull out of the water into the air, drag on the hull at any given speed is reduced $\approx 800$ times; only the wings need suffer water resistance as opposed to air resistance. (We define "hull" as incorporating all parts of a hydrofoil that are lifted out of the water, e.g., including most or all of the struts.) Thus, probably uniquely among aircraft, for hydrofoils two values of aerodynamic-medium density $\rho$ are pertinent: $\rho_{\text {drag }}$, the density pertinent to drag, and $\rho_{\text {lift }}$, the density pertinent to lift. Also, probably uniquely among aircraft other than hovercraft, for hydrofoils unless there is neither wind nor water current two values of velocity $v$ are pertinent: $v_{\text {drag }}$, the velocity pertinent to drag, ${ }^{19}$ Our knowledge of Mars is increasing rapidly. See in addition to Ref. [38] itself relevant articles that cite Ref. [38]. For an overview, see the following article (most recently revised in 2019) at https://www.wikipedia.org: "Mars". 
and $v_{\text {lift }}$, the velocity pertinent to lift. Let $\rho_{\text {air }}$ be the density of air, $\rho_{\text {water }}$ be the density of water, $v_{\text {air }}$ be the velocity of a hydrofoil relative to the air, $v_{\text {water }}$ be the velocity of a hydrofoil relative to the water, $\left(\rho v^{2}\right)_{\text {air }}=\rho_{\text {air }} v_{\text {air }}^{2}$, and $\left(\rho v^{2}\right)_{\text {water }}=\rho_{\text {water }} v_{\text {water }}^{2}$. Drag on a hydrofoil probably can be construed most simply via $\left\langle\rho v^{2}\right\rangle_{\text {drag }}$, the average with respect to drag of $\rho v^{2}$, as follows:

$$
\begin{aligned}
\left\langle\rho v^{2}\right\rangle_{\text {drag }} & =\frac{A_{\text {frontal,eff,hull }}\left(\rho v^{2}\right)_{\text {air }}+A_{\text {frontal,eff,wing }}\left(\rho v^{2}\right)_{\text {water }}}{A_{\text {frontal,eff,hull }}+A_{\text {frontal,eff,wing }}} \\
& =\frac{A_{\text {frontal,eff,hull }}\left(\rho v^{2}\right)_{\text {air }}+A_{\text {frontal,eff,wing }}\left(\rho v^{2}\right)_{\text {water }}}{A_{\text {frontal,eff,total }}} .
\end{aligned}
$$

This average value $\left\langle\rho v^{2}\right\rangle_{\text {drag }}$ can be employed in Equation (7). Note that, in Equation (18), $A_{\text {frontal,eff,wing }}$ is the effective frontal cross-sectional area of a hydrofoil's wings with respect to $d r a g$ as per the first two paragraphs of Section 3.1.1, not the effective surface area of its wings with respect to lift as per the third and fourth paragraphs of Section 3.1.1. Since typical speeds of hydrofoils are $\sim 5$ times typical wind speeds, ${ }^{20} v_{\text {water }}$ and $v_{\text {air }}$ by and large differ by $\sim 20 \%$. (Water currents are usually much slower than winds.) In contrast with Equation (7) for drag, Equation (9) for lift requires no reinterpretation for hydrofoils other than setting $\rho=\rho_{\text {water }}$ and $v=v_{\text {water }}$.

Because water is $\approx 800$ times as dense as air at sea level, the wings of a hydrofoil need only have $\approx 1 / 800$ of the surface area with respect to lift as the wings of a (low-speed) airplane in order to obtain the same lift (if $C_{L}$ is the same for both the hydrofoil and the airplane, and if $v_{\text {water }}$ for the hydrofoil equals $v_{\text {air }}$ for the airplane).

Any surface watercraft experiences an additional form of drag that we do not consider in this paper: wave drag, the energy cost of generating waves (of course not to be confused with shock-wave drag experienced by aircraft at and in the vicinity of Mach 1). ${ }^{21}$ But, because only the wings and at most only the lower part of the struts of a hydrofoil, which have minimal surface area, intersect the surface of the water, this form of drag is minimal for a hydrofoil if, as we assume, it cruises at sufficient speed to lift all but its wings and at most the lower part of its struts completely out of the water.

For hydrofoils, as for other aircraft, the energy cost $E_{A}$ for traversing given horizontal distance $X$ is directly proportional to $m g$. Hence reducing $m g$ reduces the energy cost of aerodynamic level hydrofoil flight traversing given horizontal distance $X$ equally and in direct proportion to the reduction in $m g$. Thus, for example, since $g$ on Mars is $\approx 0.38$ of $g$ on Earth, if atmospheric pressure on Mars were high enough for liquid water to exist, then $E_{A, \text { Mars }}$ for aerodynamic level flight of a hydrofoil of given mass $m$ traversing given horizontal distance $X$ on Mars would be $\approx 0.38$ of $E_{A \text {,Earth }}$ required on Earth. (As mentioned in the second paragraph of Section 3.3.2, atmospheric pressure on Mars was high enough for liquid water to exist in the past [37], but perhaps by a smaller margin than generally believed [38]. ${ }^{19}$ ) 


\subsection{The Optimum Range of Air Densities for Aerodynamic Level Flight}

Hydrofoils excepted, the range of aerodynamic-medium (air) densities on Earth, from $\approx 1 \mathrm{~kg} / \mathrm{m}^{3}$ at sea level to $\approx 10^{-3} \mathrm{~kg} / \mathrm{m}^{3}$ at the approximate practical (as opposed to ultimate) high-altitude limit of aerodynamic level flight, seems to be optimum for aerodynamic level flight, indeed for any aerodynamic flight. Much denser aerodynamic media, such as water in the case of underwater airplanes discussed in Section 3.3.2, allow aerodynamic level flight with much less power, but owing to the great resistance of a very dense medium such as water the speed of aerodynamic level flight will then be relatively slow. (Even hydrofoils are much slower than most airplanes.) Much more rarefied aerodynamic media allow higher speeds but entail difficulties of large required power and consequent dissipation of this power via equally large frictional aerodynamic heating, as discussed in Section 3.2 and the last paragraph of Section 3.3.1. As discussed in Section 3.3.1, lower $g$ such as on Mars reduces the practical low-density limit somewhat from $\approx 10^{-3} \mathrm{~kg} / \mathrm{m}^{3}$ as obtains on Earth. But if $g$ is too small, appreciably smaller than on Mars, then an atmosphere cannot be retained at all, thus precluding aerodynamic level flight, indeed precluding any aerodynamic flight (except in pressurized indoor facilities).

The considerations of the immediately preceding paragraph are modified in the case of hydrofoils, because they obtain lift via a dense medium (water) but the vast majority of their frontal cross-sectional area (both geometrical and effective) suffers resistance or drag only from a much more rarefied one (air). Of course, if $g$ is too small, then an atmosphere cannot be retained, and with vanishing atmospheric pressure liquid water-indeed any liquid-cannot exist (except in pressurized indoor facilities).

\section{Farther against the Wind Than with it}

All flights of hand-thrown projectiles that are unpowered except for the initial throw are obviously short flights as defined in Section 2.1. Thus it is not surprising that the record traversed horizontal distances for hand-thrown projectiles obtain for those executing aerodynamic flight, e.g., Frisbees, Aerobies, and boomerangs, as opposed to those executing flight that is at least primarily ballistic, e.g., sports balls [23] [24] [25] [26] [39] [40] and even more so javelins [41] (see also Supplementary Note 15). For all flights of hand-thrown projectiles: (a) the flight distance assumes that the flight is above a horizontal (level) surface, most typically level ground but possibly a smooth water surface, and (b) the flight distance is taken as the total horizontal distance traversed along the flight path, not the straight-line distance between the beginning and ending points of the flight. The latter can be short (or even zero) even for a long return-boomerang flight.

From among hand-thrown projectiles, we define as hand-thrown aircraft

\footnotetext{
${ }^{20}$ See the following articles (all most recently revised in 2019) at https://www.wikipedia.org: "Hydrofoil", "Human-powered hydrofoil", "Sailing hydrofoil", "Vestas Sailrocket", and “America’s Cup". ${ }^{21}$ See Ref. [2], pp. 103-108 and Ref. [11], Sect. 7.9.
} 
those (e.g., Frisbees, Aerobies, and boomerangs) that are capable of aerodynamic flight—of flight with aerodynamic lift exceeding weight—at achievable throwing speeds. The record horizontal flight distances for hand-thrown aircraft as of this writing include $427.2 \mathrm{~m}=1402 \mathrm{ft}$ for boomerangs, $406.3 \mathrm{~m}=1333 \mathrm{ft}$ for Aerobies, and $338.00 \mathrm{~m}=1108 \mathrm{ft} 11.1$ in for Frisbees. Moreover (unlike primarily ballistic hand-thrown projectiles) aerodynamic Frisbees, Aerobies, and boomerangs can-since lift exceeds weight at achievable throwing speeds-traverse longer distances against the wind than with it. Hand-thrown projectiles such as sports balls (e.g., golf balls, baseballs, etc.) [23] [24] [25] [26] [39] [40] and to a lesser extent javelins [41] can obtain some help from aerodynamics (see also Supplementary Note 15). But their flights are at least primarily ballistic because, at achievable throwing speeds, aerodynamic lift cannot equal, let alone exceed, weight. Record hand-thrown flight distances as of this writing are $170 \mathrm{yd}=510 \mathrm{ft}=155 \frac{1}{2} \mathrm{~m}$ for golf balls, $445 \mathrm{ft} 10 \mathrm{in}=135.89 \mathrm{~m}$ for baseballs, and $104.80 \mathrm{~m}=343 \mathrm{ft} 9 \frac{3}{4}$ in for javelins. Sports balls (e.g., golf balls, baseballs, etc.) can achieve, via the Magnus effect ${ }^{22}$, more aerodynamic lift than javelins, though still not nearly enough to equal, let alone exceed, their weights at achievable throwing speeds. Hence the hand-thrown distance records even for sports balls, while exceeding those for javelins, still fall far short of those achieved by Frisbees, let alone by Aerobies and boomerangs. And hence also javelins and even sports balls cannot in any case traverse longer distances against the wind than with it.

We should also mention the discus [42] [43] and hand-thrown (e.g., paper or balsa) gliders [44]. The discus is a hand-thrown aircraft, whose aerodynamic lift can equal or exceed its weight at achievable throwing speeds [42] [43], even though because discuses are considerably more massive than Frisbees, Aerobies, and boomerangs their distance records are much less. Indeed their distance records are less than those of sports balls and even of javelins. As of this writing, the official discus-throw flight-distance records are $74.08 \mathrm{~m}=243 \mathrm{ft} 0 \frac{1}{2}$ in for the men's 2-kg-discus throw and $76.80 \mathrm{~m}=251 \mathrm{ft} 11 \frac{1}{2}$ in for the women's 1-kg-discus throw; the unofficial discus-throw flight-distance record is $78.14 \mathrm{~m}$ $=256 \mathrm{ft} 4.4$ in for the women's 1-kg-discus throw. The discus, like the Frisbee, Aerobie, and boomerang, can traverse longer distances against the wind than with it [42] [43]. (See also Supplementary Note 15.) Hand-thrown (e.g., paper or balsa) gliders are hand-thrown aircraft, whose aerodynamic lift can equal or exceed weight at achievable throwing speeds [44]. As of this writing, the official distance record for paper gliders is $226 \mathrm{ft} 10 \mathrm{in}=69.14 \mathrm{~m}$ [44]. Flight distances of discuses are limited by their large masses; flight distances of paper gliders are limited by their small masses and hence their small inertias. [Note: We use the term "paper glider" rather than "paper airplane" (or "paper plane" for short) 
because such hand-thrown aircraft are unpowered except for the initial throw and hence are gliders, not airplanes.]

We now describe what may be the simplest example of how a hand-thrown aircraft capable of flight with aerodynamic lift exceeding weight at achievable throwing speeds, such as a discus, Frisbee, Aerobie, or boomerang, can maintain its altitude farther if thrown horizontally against the wind than with it. Let a discus, Frisbee, Aerobie, or boomerang of mass $m$ and weight $m g$ be thrown horizontally in calm air $\left(V_{\text {wind }}=0\right)$ at altitude $h_{A, 0}$ and at ground speed $v_{\text {ground }}=v_{\text {air,min }}$, the minimum airspeed required for aerodynamic lift $L$ to equal its weight $m g$ at the angle of attack at which it is thrown. Since the air is calm, its airspeed $v_{\text {air }}$ also equals $v_{\text {air,min }}$ at the instant of being thrown. Thus with calm air at the instant of being thrown $v_{\text {air }}=v_{\text {ground }}=v_{\text {air,min }}$. Reiterating, at the instant of being thrown aerodynamic lift $L$ equals its weight $m g$ at the angle of attack at which it is thrown, i.e., $L=m g$ at this angle of attack: thus $v_{\text {airmin }}$ is the minimum airspeed required for aerodynamic level flight at this angle of attack. (To avoid confusion, in this paragraph and the next we employ subscripts to distinguish between $v_{\text {air }}, v_{\text {ground }}$, and $v_{\text {wind }}$.) [For maximum horizontal flight distance, the angle of attack must of course be that which maximizes $C_{L} / C_{D}$ and hence $L / D$. With increased angle of attack (up to a stall) $C_{L}$ increases and hence $L=m g$ obtains at a slower $v_{\text {air,min }}$. But at these increased angles of attack $C_{D}$ increases faster with increasing angle of attack than $C_{L}$, so $C_{L} / C_{D}$ and thus $L / D$ decreases, and hence horizontal flight distance is diminished.] Since a hand-thrown aircraft such as a discus, Frisbee, Aerobie, or boomerang is unpowered except for the initial throw, immediately after being thrown aerodynamic drag $D$ will have reduced $v_{\text {air }}$ (and given calm air also $v_{\text {ground }}$ ) to less than $v_{\text {air,min }}$ and hence lift $L$ to less than weight $m g$. Thus immediately after being thrown it begins to lose altitude. Thus it can maintain altitude $h_{A}=h_{A, 0}$ only at the very instant when it is thrown and hence for only infinitesimal horizontal distance $X=0$. Now let the discus, Frisbee, Aerobie, or boomerang be thrown horizontally at altitude $h_{A, 0}$ and at ground speed $v_{\text {ground }}=v_{\text {air,min }} \geq v_{\text {wind }}$ with a tail wind of speed $v_{\text {wind }}$. (The restriction to the speed range $v_{\text {ground }}=v_{\text {air,min }} \geq v_{\text {wind }}$ is for simplicity, so that we can focus on the main points. The speed range $0 \leq v_{\text {ground }}=v_{\text {air, min }}<v_{\text {wind }}$ is discussed in Supplementary Note 16.) Thus at the instant of being thrown its airspeed is $v_{\text {air }}=v_{\text {ground }}-v_{\text {wind }}=v_{\text {air,min }}-v_{\text {wind }}<v_{\text {air,min }}$. Thus even at the instant of being thrown $L<m g$. Thus it cannot maintain altitude $h_{A}=h_{A, 0}$ even at the instant of being thrown and hence not even for infinitesimal horizontal distance $X=0$. Now let the discus, Frisbee, Aerobie, or boomerang be thrown horizontally at altitude $h_{A, 0}$ at and at ground speed $v_{\text {ground }}=v_{\text {air,min }}$ against a head wind of speed $v_{\text {wind }}$. At the instant of being thrown its airspeed is $v_{\text {air }}=v_{\text {air,min }}+v_{\text {wind }}>v_{\text {air,min }}$. Thus at the instant of being thrown $L>m g$. Thus it gains altitude until drag $D$ reduces $v_{\text {air }}$ to $v_{\text {air,min }}$ and hence $L$ to $m g$, by ${ }^{22}$ See Ref. [9], pp. 31-40; and the following article (most recently revised in 2019) at https://www.wikipedia.org: "Magnus effect". 
which time it will have ascended to its peak altitude $h_{A, \text { peak }}>h_{A, 0}$. Thereafter $L<m g$ and it begins to lose altitude, soon descending past $h_{A, 0}$. But it will have traversed finite horizontal distance $X>0$ while maintaining altitude $h_{A}>h_{A, 0}$ and attaining peak altitude $h_{A, \text { peak }}>h_{A, 0}$ if thrown horizontally at $v_{\text {ground }}=v_{\text {air,min }}$ against the wind, as opposed to infinitesimal horizontal distance $X=0$ and even that barely at altitude $h_{A}=h_{A, 0}$ if thrown horizontally at $v_{\text {ground }}=v_{\text {air,min }}$ with no wind, and not even infinitesimal horizontal distance $X=0 \quad$ barely at altitude $h_{A}=h_{A, 0}$ if thrown horizontally at $v_{\text {ground }}=v_{\text {air,min }} \geq v_{\text {wind }}$ with the wind. By Section 3.1.1, especially Equations (7) and (8) and the associated discussions, typically $D$ is greater for a discus, Frisbee, Aerobie, or boomerang thrown against the wind than with it by a ratio $\mathbb{R}_{\text {drag }} \approx\left[\left(v_{\text {ground }}+v_{\text {wind }}\right) \div\left(v_{\text {ground }}-v_{\text {wind }}\right)\right]^{2}$. But $\mathbb{R}_{\text {drag }}$ is a finite number, typically only moderately larger than unity. Moreover, by Equations (9) and (10) and the associated discussions, typically $L$ is also greater for a discus, Frisbee, Aerobie, or boomerang thrown against the wind than with it by a comparable ratio $\mathbb{R}_{\text {lift }} \approx\left[\left(v_{\text {ground }}+v_{\text {wind }}\right) \div\left(v_{\text {ground }}-v_{\text {wind }}\right)\right]^{2}$. Therefore typically $L / D$ and hence $C_{L} / C_{D}$ is at least approximately equal with the wind and against the wind (and also with no wind). Hence $\mathbb{R}_{\text {drag }}$ typically being moderately larger than unity does not contravene our result that it is possible for a discus, Frisbee, Aerobie, or boomerang to maintain altitude farther against the wind than with it. In our specific examples, altitude is maintained for finite horizontal distance $X>0$ against the wind, as opposed to infinitesimal horizontal distance $X=0$ with no wind and not even infinitesimal horizontal distance $X=0$ with the wind - in all three cases the initial horizontal throw being at $v_{\text {ground }}=v_{\text {air,min }}$. Indeed, untypically [23] [24] [25] [26], as per Item (b) in the first paragraph of Section 3.1.2, $\mathbb{R}_{\text {drag }}$ can be smaller than unity. Since, by Equations (9) and (10) and the associated discussions, $\mathbb{R}_{\text {lift }}$ is always greater than unity, untypically [23] [24] [25] [26] $L / D$ and hence $C_{L} / C_{D}$ can be enhanced both via increased $L$ and via decreased $D$ against the wind as opposed to with it. (See also Supplementary Note 11.)

Two auxiliary points: (i) The scenario in the immediately preceding paragraph, of a discus, Frisbee, Aerobie, or boomerang thrown at $v_{\text {ground }}=v_{\text {air }}=v_{\text {air,min }}$, i.e., corresponding to $L=m g$, at a given angle of attack given calm air, was chosen for simplicity. But of course discuses, Frisbees, Aerobies, and boomerangs can easily be thrown considerably faster than this. They can easily be thrown fast enough so that $L>m g$ in calm air, i.e., at $v_{\text {ground }}=v_{\text {air }}>v_{\text {air,min }}$, or even so that $L>m g$ with a light or moderate tail wind, i.e., at $v_{\text {ground }}>v_{\text {wind }}+v_{\text {air,min }} \Leftrightarrow v_{\text {air }}=v_{\text {ground }}-v_{\text {wind }}>v_{\text {air,min }}$. (ii) If the wind speed exceeds maximum achievable throwing speeds, then a discus, Frisbee, Aerobie, or boomerang cannot maintain altitude farther against the wind than with it-at

${ }^{23}$ See the following websites: "Saffir-Simpson Hurricane Wind Scale" [Updated 2 January 2019 to include central North Pacific examples: left-click on "About the Saffir-Simpson Hurricane Wind Scale (PDF)"] at https://www.nhc.noaa.gov/aboutsshws.php, and "Enhanced F Scale for Tornado Damage" (Update implemented on 1 February 2007) at https://www.spc.noaa.gov/faq/tornado/ef-scale.html. 
least not relative to the ground. For example, consider throwing a discus, Frisbee, Aerobie, or boomerang into a Category 5 extreme-hurricane-force or an EF5 extreme-tornado-force head wind..$^{23}$ It will still traverse finite horizontal distance $X>0$ at altitude $h_{A}>h_{A, 0}$ (peaking at altitude $h_{A, \text { peak }}>h_{A, 0}$ ) if thrown horizontally against the wind-but relative to the air, not relative to the ground. Such an extreme wind will reverse the hand-thrown aircraft's direction of motion relative to the ground almost instantaneously, well before it traverses this finite horizontal distance $X>0$ (peaking at altitude $h_{A, \text { peak }}>h_{A, 0}$ ) relative to the ground.

By contrast, hand-thrown projectiles whose flights are primarily ballistic, such as javelins [41] or even sports balls (e.g., golf balls, baseballs, etc.) [23] [24] [25] [26] [39] [40], cannot maintain altitude farther against the wind than with it at achievable throwing speeds, irrespective of wind speed. For upon such primarily-ballistic hand-thrown projectiles, air imposes drag but, at achievable throwing speeds, provides at best insufficient lift to equal, let alone exceed, weight and perhaps in some cases no lift at all (see also Supplementary Note 15).

Thus far in this Section 4, we considered hand-thrown projectiles on Earth. Let us now briefly consider them on Mars. Maximum achievable throwing speeds are the same on Mars as on Earth, $g$ on Mars is $\approx 0.38$ of $g$ on Earth, and low-altitude air density on Mars is $\approx 1 / 70$ of that on Earth [35] [36] [37] (see also Supplementary Note 14). Thus, by the first line of Equation (14), required throwing speeds for aerodynamic flight of hand-thrown projectiles, i.e., for aerodynamic lift $L$ to equal projectile weight, on Mars are

$\approx[0.38 \div(1 / 70)]^{1 / 2}=(0.38 \times 70)^{1 / 2} \approx 5.2$ times that for identical hand-thrown projectiles on Earth. Thus aerodynamic flight of hand-thrown discuses, Frisbees, Aerobies, and boomerangs is at the very least much more difficult to achieve on Mars than on Earth, and may be impossible to achieve on Mars. In order for low-altitude achievable throwing speeds of hand-thrown discuses, Frisbees, Aerobies, and boomerangs on Mars to equal those on Earth, by the first line of Equation (14) their masses would have to be reduced by a factor of $\approx 0.38 \div(1 / 70)=0.38 \times 70 \approx 27$ while still maintaining their same aerodynamic lifting areas and hence their same sizes and shapes as on Earth. Thus, with respect to hand-thrown aircraft, lower $g$ on Mars is insufficient to compensate for lower air density there.

\section{Energy Efficiency of Surface Transportation versus That of Flight}

It may be of interest to compare the energy efficiency of both aerodynamic level flight and ballistic flight with that of horizontal surface (land and/or water) transportation. If the frictional force opposing horizontal motion of a surface vehicle of mass $m$ is a fraction $F$ of its weight $m g$, then the energy cost of its traversing horizontal distance $X$ is

$$
E_{S}=m g F X .
$$


(The subscript $S$ denotes surface transportation.)

Thus $F$ plays the same role in surface transportation that $D / L$ plays in aerodynamic level flight, or, equivalently, $1 / F$ plays the same role in surface transportation that $L / D$ plays in aerodynamic level flight. For one circumnavigation at Earth's surface $X=2 \pi R$ and $E_{S}=2 \pi R m g F$; for $N$ circumnavigations at Earth's surface $X=2 \pi R N$ and $E_{S}=2 \pi R m g F N$. (A single-circumnavigation journey is the longest possible one whose purpose is to reach a destination on Earth, with the destination being the starting point after traveling around the world.)

Typical values of the coefficient of surface friction $C_{S}$ for land vehicles range from $\approx 0.01$ to $\approx 1$ for sliding friction ( $\approx 0.005$ for some maglev trains), and as low as $\approx 0.001$ for rolling friction of hard wheels on hard surfaces-for example low-speed to moderate-speed traditional (not maglev) railroad transportation. For land vehicles at speeds low enough that air resistance is small compared to surface (e.g., sliding, rolling, or maglev) friction, $F=C_{S}$ and is at least approximately independent of speed. For land vehicles at higher speeds $F=C_{S}+D / m g, D$ being given by Equation (7) with $\rho$ being the density of air. (While traditional railroad transportation is more energy-efficient at low to moderate speeds, maglev trains almost completely abolish wear on the tracks, owing to their lack of mechanical contact with the tracks.) See Supplementary Note 17.

Since even minimum-altitude circular-orbit ballistic spaceflight must be above any appreciable atmosphere, $r_{B}$ for even minimum-altitude circular-orbit ballistic spaceflight must exceed $R$. But for simplicity, as in Section 2.2, we let the orbit be a minimum-altitude circular one, for which $h_{B}=r_{B}-R \ll R$ and hence can be neglected in comparison with $R$. (For surface transportation at sea level $h_{S}=0$ and hence $r_{S}=R$.) Identically as in the case of aerodynamic flight, in order for surface transportation to be more energy-efficient than ballistic transportation, neglecting air resistance in the latter, we require $F<1 / 2$ for short journeys $(X \ll 2 \pi R$ ) and $F<1 / 4 \pi$ for single-circumnavigation journeys $(X=2 \pi R)$. For journeys of intermediate length ( $X$ ranging from much smaller than $R$ to approaching $2 \pi R$ ), the value that $F$ cannot equal or exceed if surface transportation is to be more energy-efficient than ballistic transportation, neglecting air resistance in the latter, decreases monotonically from $1 / 2$ towards $1 / 4 \pi$ as $X$ increases from very small values towards $2 \pi R$. (Since if $X=2 \pi R$ minimum-energy ballistic spaceflight is a circular orbit just above appreciable atmosphere at altitude $h_{B}$, it cannot begin and end at the altitude $h_{s}$ of surface transportation, but $h_{B}-h_{s} \ll R$.)

The requirement $F<1 / 2$ for short $(X \ll 2 \pi R$ ) surface journeys to be more energy-efficient than short ballistic journeys neglects air resistance in ballistic flight. But air resistance is not always negligible in short ballistic flights, especially in the lower atmosphere. If it is not neglected, then the requirement is weakened to $F<1 / 2 n$ with $n<1$. The requirement $F<4 \pi$ for single-circumnavigation surface journeys to be more energy-efficient than single-circumnavigation 
minimum-altitude circular-orbit ballistic journeys neglects air resistance in minimum-altitude circular-orbit ballistic spaceflight; if it is not neglected, then the requirement is weakened to $F<1 / 4 \pi n$ with $n<1$. But it is weakened only very slightly, because air resistance even at minimum-circular-orbit spaceflight altitude is very small. Not neglecting air resistance in ballistic flight, for journeys of intermediate length ( $X$ ranging from much smaller than $R$ to approaching $2 \pi R$ ), the value that $F$ cannot equal or exceed if surface transportation is to be more energy-efficient than ballistic flight decreases monotonically from $1 / 2 n(X)$ towards $1 / 4 \pi n(X)$ as $X$ increases from very small values towards $2 \pi R: n(X)<1$ but increases monotonically towards very nearly 1 as $X$ increases from very small values towards $2 \pi R$. Air resistance in minimum-altitude circular-orbit ballistic flight is very small and hence also $1-n(X)$ is very small, i.e., $n(X)$ is very nearly 1 , if $X=2 \pi R$. (Since then minimum-energy ballistic spaceflight is a circular orbit just above appreciable atmosphere at altitude $h_{B}$, it hence cannot begin and end at the altitude $h_{S}$ of surface transportation, but $h_{B}-h_{S} \ll R$.)

By Equation (19) the energy cost $E_{S}$ of surface transportation increases linearly with increasing $X$ and hence for $N$-circumnavigation journeys also with increasing $N$, identically as with aerodynamic flight by Equation (2) and Section 2.3. By contrast, for minimum-altitude circular-orbit ballistic spaceflight, irrespective of $X$ and hence also of $N, E_{B}$ remains fixed at the value given by Equation (3) for $N=1$. For, even at minimum-circular-orbit altitude, air resistance is almost negligible, i.e., space is almost frictionless; thus the energy cost of launching a spacecraft is one-time. Thus for multi-circumnavigation ( $X=2 \pi R N, N \gg 1$ ) journeys, the energy efficiency of even minimum-altitude circular-orbit ballistic spaceflight surpasses that of surface transportation by an arbitrarily large margin, just as it does that of aerodynamic flight by an arbitrarily large margin, the margin being even larger if spaceflight is high-orbit and even larger yet if it exceeds escape velocity. With respect to both aerodynamic flight and surface transportation the reason is that stated in Section 2.3: Space is essentially frictionless, and increasingly frictionless with increasing altitude, thus allowing spacecraft but neither aircraft nor surface vehicles to take full advantage of Newton's first law of motion (inertia). ${ }^{5}$ The energy cost of speed in spaceflight is one-time, the energy cost of speed in aerodynamic flight and in surface transportation is never-ending. ${ }^{5}$ Spaceflight is thus the only mode of transportation that can achieve $\infty \mathrm{mi} / \mathrm{gal}=\infty \mathrm{km} / \mathrm{l}$ of fuel (or the equivalent thereof)Spaceship Earth (whose fuel for its orbital and rotational motions was part of the solar nebula's kinetic energy) is a good example. ${ }^{5}$ To save time in spaceflight continuous energy expenditure can be employed, for example employing solar, laser, or on-board nuclear energy. But in spaceflight continuous energy expenditure buys acceleration; in aerodynamic flight and in surface transportation it buys only (constant) speed. ${ }^{5}$

We note that lighter-than-air craft, for example dirigibles and blimps, should 
be included within the category of surface transportation rather than within the category of aerodynamic flight, because their lift obtains typically at least primarily and often entirely via buoyancy rather than via aerodynamics. Similarly the lift for surface (land and/or water) transportation vehicles obtains via support of the ground for land surface vehicles and via buoyancy for ships and submarines, rather than via aerodynamics. In this regard, we construe maglev and air-cushion vehicles as being surface (land and/or water) transportation vehicles rather than aircraft, because their lift obtains via support of the surface through the intermediary of a magnetic-repulsion or an air cushion. [Aircraft very near the ground obtain some extra lift from the air-cushion "ground effect" (see Supplementary Note 18).] For a dirigible or blimp in level flight $F$ is the ratio of air resistance to the unbuoyed weight of the dirigible or blimp, i.e., $F=D / m g, D$ being given by Equation (7) with $\rho$ being the density of air at flight altitude. For a fully-submerged submarine $F$ is the ratio of water resistance to the unbuoyed weight of the submarine, i.e., $F=D / m g, D$ being given by Equation (7) with $\rho$ being the density of water. For a ship or surface-cruising submarine, or for a hydrofoil, $F$ is the ratio of combined water and air resistance to the unbuoyed weight of the vehicle, i.e., $F=D / m g, D$ being given by Equation (7) with $\left\langle\rho v^{2}\right\rangle_{\text {drag }}$ taken as for hydrofoils as per Section 3.4 [see especially Equation (18) and the associated discussions]. ( $F$ for land vehicles was discussed in the third paragraph of this Section 5.) By contrast, as previously noted [see especially Section 3.4 but also the second paragraph of Section 1, the second paragraph following that containing Equation (2), and Section 3.5], hydrofoils should be classified as aircraft, because their lift obtains primarily if not essentially entirely aerodynamically rather than via buoyancy, even though the density $\rho$ of their aerodynamic medium (water) is $\approx 800$ times that of air at sea level. (By lifting the hull out of water into air, drag on the hull at any given speed is reduced $\approx 800$ times; only the wings need suffer water resistance as opposed to air resistance.) Any surface-cruising watercraft experiences wave drag, i.e., the energy cost of generating waves (of course not to be confused with shock-wave drag experienced by aircraft at and in the vicinity of Mach 1$),{ }^{21}$ which we have not considered in this paper. Wave drag is minimal for a hydrofoil cruising at sufficient speed to lift its hull completely out of the water, but for ships and surface-cruising submarines it is typically the largest component of drag. ${ }^{21}$

While this is obvious, perhaps it is worthwhile to note that there is a minimum flight speed for any (nonhovering) aircraft, but no minimum speed for land vehicles, dirigibles, ships, or submarines. Increased induced drag is imposed on (nonhovering) aircraft at minimum flight speed (see Supplementary Notes 6 and 9). By contrast: (a) For land vehicles, as speed is reduced to zero, $F=C_{S}$ remains at least approximately constant. (b) For dirigibles, for ships, and for both fully-submerged and surface-cruising submarines, as speed is reduced to zero, $D$ and hence also $F=D / m g$ is reduced to zero.

Of course, the remarks of the last paragraph of Section 2.1, and especially the remarks of Section 2.4, distinguishing between the energy efficiency of aerody- 
namic or ballistic flight per se and the energy efficiency of the engine that powers aerodynamic or ballistic flight apply equally in distinguishing between the energy efficiency of surface transportation per se and the energy efficiency of the engine that powers surface transportation. The energy that must be supplied to an engine whose efficiency is $\epsilon$ in order to facilitate surface transportation requiring energy $E_{S}$ as per Equation (19) is of course $E_{S} / \epsilon$.

Generalizing the third-to-last paragraph of Section 2.1 and the last paragraph of Section 2.2 in light of this Section 5, if air resistance in ballistic flight and in surface transportation can be neglected, then for traversal of any given horizontal distance $X$, short or long, all three quantities $E_{B}, E_{A}$, and $E_{S}$ are directly proportional to $m g$. Hence reducing $m g$ reduces the energy cost of ballistic flight, aerodynamic level flight, and surface transportation traversing any given horizontal distance $X$ equally and in direct proportion to the reduction in $\mathrm{mg}$, but does not alter the ratio of energy costs between these three modes of transportation. If air resistance in ballistic flight or in surface transportation cannot be neglected, then reducing $m g$ reduces the energy cost of ballistic flight or of surface transportation, respectively, less than in direct proportion to the reduction in $\mathrm{mg}$.

Generalizing the second-to-last paragraph of Section 2.1 in light of this Section 5 , most typically, $m g$ is reduced by reducing $m$. But we can also consider reduction of $g$. Two examples: (i) Aerodynamic and ballistic flight, as well as surface transportation, on Mars is at lower $g$. (ii) An aircraft, watercraft, or surface vehicle of mass $m$ a fraction $f(0<f<1)$ of whose weight $m g$ is offset by buoyancy can be construed as either being of effective mass $m(1-f)$ in a gravitational field $g$ or as being of mass $m$ in a gravitational field of effective strength $g(1-f)$. Such partial offset of weight by buoyancy obtains, for example, for a dirigible or blimp that relies on buoyancy for only part of its lift, with the balance obtaining aerodynamically, for a hydrofoil that cruises so slowly that it must rely on buoyancy for part of its lift, or for an underwater surface vehicle that is denser than water. Because all solids are hundreds to thousands of times as dense as air at sea level, $f$ is negligible for surface vehicles on land as it is for aerodynamic vehicles (aircraft) in air. But $f$ is not negligible for underwater surface vehicles (and for underwater airplanes as discussed in Section 3.3.2) that are denser than water, because even the densest solids are little more than 20 times as dense as water. [An evenhanded comparison, in Section 3.3.2, between a standard (atmospheric) airplane's aerodynamic level flight in air and an underwater airplane's aerodynamic level flight in water was facilitated by an extra $10^{3}$ $\mathrm{kg} / \mathrm{m}^{3}$ of density for the latter to offset the buoyancy provided by water.]

In this paper in general we have not considered the energy cost of building and maintaining vehicles. In this Section 5 in particular we also have not considered the energy cost of building and maintaining pathways for surface transportation. Concerning the latter, we have not, for example, considered the energy cost of building and maintaining roads, railroads, and canals. Of course, 
for transportation that does not require artificially-built pathways, such as transportation in air, on or in water (except via canal), on land and/or water via hovercraft or other surface vehicles that do not require roads, or via spaceflight, this latter energy cost is zero.

\section{Brief Concluding Remarks}

Hopefully, we have provided at least somewhat helpful insights concerning energy efficiency in aerodynamic versus ballistic flight, concerning aerodynamic lift and drag, concerning selected aspects and examples of flight, in distinguishing between the energy efficiency of flight per se and the energy efficiency of the engine that powers flight, and via considering the relation between the density of an aerodynamic medium and aerodynamic level flight. Also, hopefully, our comparison with the energy efficiency of surface transportation and our discussion of surface transportation have been helpful.

While we have focused mostly on Earth, with some consideration of Mars, our results are easily generalizable to any planet or other astronomical bodies on which aerodynamic flight and/or surface travel is possible, i.e., to any planet or other astronomical bodies with an atmosphere, and/or a solid and/or liquid surface. Also, they are valid irrespective of the values of $M, R, g, m, \rho$, and (except as for simplicity we assume $\left.h_{A}<h_{B} \ll R\right)$ of $h_{A}, r_{A}=R+h_{A}, h_{B}$, and $r_{B}=R+h_{B}$ [2]-[15]

We should emphasize the limitations of this present work. In this paper our main goal was to elucidate more conceptually than mathematically some fundamental ideas concerning energy efficiency and a number of other aspects of aerodynamic versus ballistic flight, and to provide comparison with surface transportation. We did not attempt the mathematically complex and detailed fully-quantitative analyses based on rigorous application of fluid dynamics, e.g., computational fluid dynamics, as is required in the actual design of aircraft, or the analyses required in the actual design of spacecraft or surface vehicles. We also neglected many details required in the actual operation of vehicles: to mention just one example of many, we neglected reduction of vehicle mass $m$ as fuel is consumed. Moreover, we focused mainly on the paramount $\rho v^{2}$ functional dependency of lift and drag, which is the first-order dependency upon $\rho$ and upon $v$. Considerations of departures from the first-order $\rho v^{2}$ functional dependency [27] [28], whether the large departures as per Items (a), (b), and (c) discussed in the first paragraph of Section 3.1.2, or the smaller departures that can yield the modest but still significant improvements in aircraft energy efficiency [27] [28] discussed in the second, third, and fourth paragraphs thereof and in the second paragraph of Section 3.3.1 (see also Supplementary Notes 5, 6, $8-12$, and 14), require mathematically complex and detailed fully-quantitative analyses based on rigorous application of fluid dynamics, e.g., computational fluid dynamics. Such analyses are of course essential in the actual design of aircraft $^{14}$ [2]-[15] [27] [28] and even sports balls [23] [24] [25] [26] (see also Sup- 
plementary Note 15). But we did not attempt them in this paper: our analyses were qualitative to semiquantitative. We thus gave only limited considerations of departures from the first-order $\rho v^{2}$ functional dependency of lift and drag. Thus we concealed the difficult and complex physics underlying these departures within $C_{D}$ and $C_{L}$, specifically, within departures of $C_{D}$ and $C_{L}$ from constancy. Thus again our analyses were qualitative to semiquantitative. But hopefully they may still have been helpful.

In closing, we note that, even given all of the advances in aerodynamics, new discoveries are still being made, e.g., see Ref. [46].

\section{Acknowledgements}

I thank Dr. Donald H. Kobe for very helpful discussions concerning fluid dynamics (especially aerodynamic drag), and for very insightful general scientific discussions over very many years. I am very grateful to my father Amnon Denur for giving me Refs. [13] and [34], and to my grandfather Moe Levin for giving me Ref. [14]: these three works introduced me to the science of flight in the 1960s. I also thank Robert H. Shelton and Adam Shelton for very helpful discussions several years ago concerning hand-thrown aircraft, Robert $\mathrm{H}$. Shelton for recent thought-provoking discussions concerning aerodynamics in general, focusing in particular on highly-streamlined dirigibles both in neutral-buoyancy flight and assisted by aerodynamic lift, and Robert H. Shelton also for reading this manuscript and for very helpful advice concerning diction. I am very grateful to Dr. S. Mort Zimmerman and to Dr. Stan Czamanski for very insightful general scientific discussions over many years. Additionally, I thank Dan Zimmerman, Dr. Kurt W. Hess, and Robert H. Shelton for very insightful general scientific discussions at times (especially those with Dr. Kurt W. Hess concerning fluid dynamics), including brief yet very interesting discussions relevant to this paper.

\section{Conflicts of Interest}

The author declares no conflicts of interest regarding the publication of this paper.

\section{References}

[1] Walker, J., Halliday, D. and Resnick, R. (2018) Fundamentals of Physics. 11th Edition, John Wiley \& Sons, Hoboken, NJ.

[2] Macmahon, T.A. and Bonner, J.T. (1983) On Size and Life. Scientific American Books, New York, Chapter 3-6.

[3] Adams, J.A. (2006) Mathematics in Nature, Third Printing, and First Paperback Printing, with a New Preface. Princeton University Press, Princeton, NJ.

[4] Norman, F. and Smith, N.F. (1972) Bernoulli and Newton in Fluid Mechanics. The Physics Teacher, 10, 451-455. https://doi.org/10.1119/1.2352317

[5] Weltner, K. (1987) A Comparison of Explanations of the Aerodynamic Lifting Force. American Journal of Physics, 55, 50-54. https://doi.org/10.1119/1.14960 
[6] Auerbach, D. (1988) On the Problem of Explaining Lift. American Journal of Physics, 56, 853. https://doi.org/10.1119/1.15444

[7] Weltner, K. (1988) Response to "On the Problem of Explaining Lift" [Am. J. Phys. 56, 853 (1988)]. American Journal of Physics, 56, 853. https://doi.org/10.1119/1.15445

[8] Waltham, C. (1998) Lift without Bernoulli. The Physics Teacher, 36, 457-462. https://doi.org/10.1119/1.879927

[9] von Kármán, T. (2004) Aerodynamics: Selected Topics in the Light of Their Historical Development. Dover, Mineola, New York.

[10] von Mises, R. (2004) Theory of Flight. Dover, New York.

[11] Granger, R.A. (1995) Fluid Mechanics. Dover, New York.

[12] Darlington, A. (2003) Virtuous Circle-The Development of Composite Fibre Sailplanes. Ingenia, 15, 26-29. https://www.ingenia.org.uk/Ingenia/Contents/Issue15

[13] Jones, B. (1947) Aerodynamics for Pilots. United States Government Printing Office, Washington DC.

[14] Stever, H.G. and Haggerty, J.J. (1965) Flight. Time Inc., New York, Chapter 3.

[15] Allen, R.C.S. (1969) Theory of Flight for Glider Pilots. 2nd Edition, Oliver and Boyd, Edinburgh.

[16] Bohren, C.F. and Albrecht, B.A. (1998) Atmospheric Thermodynamics. Oxford University Press, New York, 65.

[17] Wallace, J.M. and Hobbs, P.V. (2006) Atmospheric Science. 2nd Edition, Academic Press, Amsterdam.

[18] Holton, J.R. and Hakin, G.J. (2013) An Introduction to Dynamic Meteorology. 5th Edition, Academic Press, Amsterdam.

[19] Curzon, F.L. and Ahlborn, B. (1975). Efficiency of a Carnot Engine at Maximum Power Output. American Journal of Physics, 43, 22-24. https://doi.org/10.1119/1.10023

[20] Vaudrey, A., Lanzetta, F. and Feidt, M. (2014) H. B. Reitlinger and the Origins of the Efficiency at Maximum Power Formula for Heat Engines. Journal of Non-Equilibrium Thermodynamics, 39, 199-203. https://doi.org/10.1515/jnet-2014-0018

[21] McLean, D. (2005) Wingtip Devices: What They Do and How They Do It. 2015 Boeing Performance and Flight Operations Engineering Conference, Seattle, WA, 1-20. https://www.smartcockpit.com/docs/Wingtip Devices.pdf

[22] Neal, L., Harrison, N. and Mujezinovic, D. (2004) Wingtip Devices. https://www.dept.aoe.vt.edu/ mason/Mason f/WingtipDevicesS04.pdf

[23] Jargodzi, C.P. and Potter, F. (2001) Mad About Physics. John Wiley \& Sons, New York, 267-268.

[24] Erlichson, H. (1983) Maximum Projectile Range with Drag and Lift, with Particular Application to Golf. American Journal of Physics, 51, 357-362. https://doi.org/10.1119/1.13248

[25] MacDonald, W.M. and Hanzely, S. (1991) The Physics of the Drive in Golf. American Journal of Physics, 59, 213-218. https://doi.org/10.1119/1.16564

[26] Jorgensen, T.P. (1999) The Physics of Golf. 2nd Edition, Springer, New York, Chapter 8.

[27] Riehl, H. (1972) Introduction to the Atmosphere. 2nd Edition, McGraw-Hill, New 
York, Chapter 13, 435-442.

[28] Ohrn, K.E. (2007) Aircraft Energy Use. In: Capehart, B.L., Ed., Encyclopedia of Energy Engineering and Technology, CRC Press, Boca Raton, FL, 24-31.

[29] Champion, K.S.W., Cole, A.E. and Kantor, A.J. (2003) Standard and Reference Atmospheres. In: Zdyb, G.J., Ed., Handbook of Geophysics and the Space Environment, NOAA, Washington DC, Chapter 14.

https://www.cnofs/Handbook of Geophysics 1985/Chptr14.pdf

http://www.cnofs.org/Handbook of Geophysics 1985/

[30] Gringorten, I.I., Kantor, A.J., Izumi, Y., Tattelman, P.I. and Cole, A.E. (2003) Atmospheric Temperatures, Density and Pressure. In: Zdyb, G.J., Ed., Handbook of Geophysics and the Space Environment, NOAA, Washington DC, Chapter 15. https://www.cnofs/Handbook of Geophysics 1985/Chptr15.pdf http://www.cnofs.org/Handbook of Geophysics 1985/

[31] Grantham, D.D., Gringorten, I.I., Kantor, A.J., Dyer, R.M., Tattelman, P., Barnes, A.A., Bertoni, E.A., Cohen, I.D., Glass, C.M., Hardy, K.R., Izumi, Y., Metcalf, J.I., Brown, H.A. and Kunkel, B.A. (2003) Water Vapor, Precipitation, Clouds and Fog In: Zdyb, G.J., Ed., Handbook of Geophysics and the Space Environment, National Oceanic and Atmospheric Administration (NOAA), Washington DC, Chapter 16. https://www.cnofs/Handbook of Geophysics 1985/Chptr16.pdf http://www.cnofs.org/Handbook of Geophysics 1985/

[32] Champion, K.S.W., O’Sullivan, W.J. and Teweles, S. (1962) U.S. Standard Atmosphere, 1962.

https://www.ntrs.nasa.gov/archive/nasa/casi.ntrs.nasa.gov/19630003300.pdf

[33] McCartney, E.J. (1976) Optics of the Atmosphere. John Wiley \& Sons, New York.

[34] Haynes, B.C. (1943) Meteorology for Pilots. 2nd Edition, United States Government Printing Office, Washington DC, Chapter 4, 22-29.

[35] Williams, D.R. (2018) Mars Fact Sheet. NASA (National Space Science Data Center). https://nssdc.gsfc.nasa.gov/planetary/factsheet/marsfact.html

[36] Williams, D.R. (2019) Earth Fact Sheet. NASA (National Space Science Data Center). https://nssdc.gsfc.nasa.gov/planetary/factsheet/earthfact.html

[37] Malin, M.C., Belton, M.J.S. and Carr, M.H. (Note: The order of these three authors changes frequently.) (2019) Mars. https://www.britannica.com/place/Mars-planet

[38] Hu, R., Cass, D.M., Ehlmann, B.L. and Yung, Y.L. (2015) Tracing the Fate of Carbon and the Atmospheric Evolution of Mars. Nature Communications, 6, Article No. 10003. https://doi.org/10.1038/ncomms10003

[39] Nathan, A.M. (2008) The Effect of Spin on the Flight of a Baseball. American Journal of Physics, 76, 119-124. https://doi.org/10.1119/1.2805242

[40] Mehta, R.D. and Pallis, J.M. (2001) Sports Ball Aerodynamics: Effects of Velocity, Spin and Surface Roughness. In: Froes, F.H. and Haake, S.J., Eds., Materials and Science in Sports, TMS, Warrendale, Pennsylvania, PA, 185-197.

[41] Bartlett, R.M. (2009) The Aerodynamics of Javelin Flight-A Re-Evaluation. https://ojs.ub.uni-konstanz.de/cpa/article/viewFile/2340/2206

[42] Seo, K., Shimoyoma, K., Ohta, K., Ohgi, Y. and Kimura, Y. (2012) Aerodynamic Behavior of a Discus. Procedia Engineering, 34, 92-97. https://doi.org/10.1016/j.proeng.2012.04.017

[43] Maheras, A. (2016) Long Distance: Basic Aerodynamics and Flight Characteristics in Discus Throwing. Techniques for Track \& Field and Cross Country, 9, 8-20. 
[44] Paper Plane (2019) https://www.wikipedia.org

[45] von Kármán, T. and Pai, S.I. (1963) From Low-Speed Aerodynamics to Astronautics. Macmillan and Pergamon, New York.

[46] Domel, A.G., Saadat, M., Weaver, J.C., Haj-Harari, H., Bertoldi, K. and Lauder, G.V. (2018) Shark Skin-Inspired Designs That Improve Aerodynamic Performance. Journal of the Royal Society Interface, 15, 201700828.

https://doi.org/10.1098/rsif.2017.0828 


\section{Appendix: Supplementary Notes}

The Supplementary Notes in this Appendix provide auxiliary information concerning topics discussed in the text and/or in the cited references.

Supplementary Note 1: A succinct discussion of aerodynamic lift and drag is provided in Chap. Thirteen of Ref. [3]. The relation of aerodynamic lift to Bernoulli's principle, which is often oversimplified in elementary textbooks, is synopsized on pp. 304-308. (Note: Lift does not become arbitrarily large with increasing arbitrarily chosen height and/or width of the near-rectangular region in Fig. 13.4 on p. 306 of Ref. [3]. If this region is arbitrarily chosen larger, then the wing occupies a smaller fraction of it, so that the average angle $\alpha$ at which the wing can deflect air within the region downward decreases in compensation.) For more detailed discussions concerning these points see Refs. [4] [5] [6] [7] [8]; relevant material in Refs. [1] [2] [9]-[15], and [45]; and the following articles: "Lift (force)", "Lift coefficient", and "Lift-to-drag ratio" (all most recently revised in 2019) at https://www.wikipedia.org, and other Wikipedia articles concerning lift that are cited therein; and "Lift" (most recently revised in 2017) at https://www.skybrary.aero/index.php/Main Page, and other SKYbrary articles concerning lift that are cited therein. [Reference [14] is included in our citations mainly as a historical source. As is the case with many books written for laymen, its explanation of lift based on Bernoulli's principle (on pp. 53-54) is an oversimplification. One element of this explanation of lift implies that air must take the same time to traverse the upper and lower surfaces of an airplane wing. This "equal-transit-time" element is incorrect: the path traversing the upper surface of an airplane wing is typically, say, $\approx 5 \%$ to $\approx 10 \%$ longer than that traversing the lower surface, but air traversing the upper surface typically can move up to $\approx 2$ times the free-stream velocity, and hence up to more than $\approx 2$ times faster than that traversing the lower surface: See Decker, J.S. (2014) See How It Flies. https://www.av8n.com/how/ (most especially Section 3.2 thereof). But otherwise Ref. [14] in general, and the explanations of aerodynamics in Chap. 3 thereof in particular, seem to be qualitatively correct. Reference [15] is included in our citations because, even though introductory, unlike our other cited references it concentrates on gliding flight and hence provides a different perspective.

Supplementary Note 2: An example of differences of viewpoint in explaining aerodynamic lift is provided in the exchange of ideas in Refs. [5] [6] [7]. (Reference [5] is cited in the discussions of Ref. [3] mentioned in Supplementary Note 1.)

Supplementary Note 3: Reference [10] probably covers considerably more material, and in considerably greater depth, than is sufficient for this present paper.

Supplementary Note 4: Information concerning the Eta glider is accessible in Ref. [12]. Additional information concerning the Eta glider and related 
gliders is accessible at https://www.leichtwerk.de. The Eta glider probably has the highest $L / D$ ratio of any sailplane built thus far. For additional related information see the following articles (all most recently revised in 2019) at https://www.wikipedia.org: "Lift-to-drag ratio", "Lift coefficient", "Drag coefficient", "Gliding flight", and "Eta (glider)".

Supplementary Note 5: The most energy-efficient angle of attack for aerodynamic flight, which maximizes $L / D$, is always assumed in this present paper unless otherwise noted. Angle of attack is defined on pp. 114-115 and 139-140 of Ref. [10], and on pp. 30-33 of Ref. [13]. Various aspects of maximization of energy efficiency of aerodynamic flight via this choice of angle of attack are discussed on pp. 172-176 and 203-209 of Ref. [2], pp. 60-67 of Ref. [9], Chap. VII of Ref. [10], Section 12.11 of Ref. [11], and Chaps. VIII, IX, and XI of Ref. [13]. Graphs of $C_{L}, C_{D}$, and $L / D$ versus angle of attack, and related discussions, are provided for dragonfly flight on pp. 206-208 of Ref. [2], for one airfoil on p. 44 of Ref. [9], for numerous airfoils in Chap. VII of Ref. [10], for one airfoil on pp. 649-651 of Ref. [11], and for a few airfoils in Chap. IV of Ref. [13].

Note that, as per Section XVI.1 of Ref. [10], and as per the simpler examples provided in Table VII on p. 75 (in Chap. VIII) and Table IX on p. 80 (in Chap. IX) of Ref. [13], the angle of attack corresponding to minimum energy cost $E_{A}$ per unit traversed distance $X$ in aerodynamic level flight is somewhat smaller, and the flight speed $v$ corresponding thereto is somewhat larger, than the values corresponding to minimum required power $P_{A}$. [Both $X$ and $v$ are relative to the air (also relative to the ground if the wind is calm).] For a faster flight traversing distance $X$ requires less time $t$ than a slower one; thus $E_{A}=P_{A} t=P_{A} X / v$ and hence $E_{A} / X=P_{A} / v$ is minimized at a somewhat larger $v$ and at a somewhat smaller angle of attack than is $P_{A}=E_{A} / t$. From among the entries in Table VII and in the first two columns of Table IX of Ref. [13] pertinent to flight at sea level, $P_{A}$ is minimized at $14.0 \mathrm{hp}$, at an angle of attack of $12^{\circ}$, and at $V=45.0 \mathrm{mi} / \mathrm{h}$, with $P_{A} / v=E_{A} / X=14.0 \mathrm{hp} /(45.0 \mathrm{mi} / \mathrm{h})=0.311 \mathrm{hp} \cdot \mathrm{h} / \mathrm{mi}$. But with $P_{A}=14.6 \mathrm{hp}$, at an angle of attack of $8^{\circ}$, and at $v=50.9 \mathrm{mi} / \mathrm{h}$, $P_{A} / v=E_{A} / X=14.6 \mathrm{hp} /(50.9 \mathrm{mi} / \mathrm{h})=0.287 \mathrm{hp} \cdot \mathrm{h} / \mathrm{mi}$, the minimum value of $P_{A} / v=E_{A} / X$ from among the tabular entries. From among the entries in the last two columns of Table IX of Ref. [13] pertinent to flight at $5000 \mathrm{ft}, P_{A}$ is minimized at $15.1 \mathrm{hp}$, at an angle of attack of $12^{\circ}$, and at $v=48.5 \mathrm{mi} / \mathrm{h}$, with $P_{A} / v=E_{A} / X=15.1 \mathrm{hp} /(48.5 \mathrm{mi} / \mathrm{h})=0.311 \mathrm{hp} \cdot \mathrm{h} / \mathrm{mi}$. But with $P_{A}=15.7 \mathrm{hp}$, at an angle of attack of $8^{\circ}$, and at $v=54.8 \mathrm{mi} / \mathrm{h}$,

$P_{A} / v=E_{A} / X=15.7 \mathrm{hp} /(54.8 \mathrm{mi} / \mathrm{h})=0.286 \mathrm{hp} \cdot \mathrm{h} / \mathrm{mi}$, the minimum value of $P_{A} / v=E_{A} / X$ from among the tabular entries. This is true in general: In aerodynamic level flight energy cost per unit distance $E_{A} / X$ is minimized and $L / D$ is maximized at a smaller angle of attack and at a higher airspeed $v$ than corresponds to minimization of $P_{A}=E_{A} / t$. Minimum required power $P_{A}=E_{A} / t$ allows an aircraft to maintain aerodynamic level flight for the max- 
imum possible time (maximum endurance); maximum energy efficiency $E_{A} / X=P_{A} / v$ (which we always assume in this present paper unless otherwise noted) allows an aircraft executing aerodynamic level flight to traverse the maximum possible distance. In gliding flight without engine power, i.e., powered only by loss of gravitational potential energy $m g h_{A}\left(P_{A}=m g\left|\mathrm{~d} h_{A}\right| / \mathrm{d} t\right)$, $E_{A} / X=m g\left|\mathrm{~d} h_{A}\right| / \mathrm{d} X$ is minimized and $L / D$ is maximized at the same angle of attack and (neglecting the weight of the fuel) at the same airspeed $v$ at which $L / D$ is maximized and $E_{A} / X=P_{A} / \nu$ is minimized in powered level flight; and sinking speed $\left|\mathrm{d} h_{A}\right| / \mathrm{d} t$ is minimized at the same angle of attack and (neglecting the weight of the fuel) at the same airspeed $v$ at which $P_{A}=E_{A} / t$ is minimized in powered level flight. Thus in gliding flight minimization of $P_{A} / v=E_{A} / X$ corresponds to maximum gliding range $X$, and minimization of $P_{A}=E_{A} / t$ corresponds to minimum sinking speed $\left|\mathrm{d} h_{A}\right| / \mathrm{d} t$, i.e., to maximum endurance or maximum time $t$ until all of the initial altitude $h_{A \text {,initial }}$ and all of the initial potential energy $m g h_{A, \text { initial }}$ have been lost. (See pp. 404-405 and Section XVI.1 of Ref. [10], and Chap. XI of Ref. [13].)

Supplementary Note 6: The most energy-efficient angle of attack, which maximizes $L / D$ and which we always assume in this present paper unless otherwise noted, is not the optimum angle of attack for all facets of flight. As per Supplementary Note 5, maximum endurance in engine-powered level flight and minimum sinking speed in gliding flight without engine power obtain at a larger angle of attack and less-than-maximum $L / D$. In this Supplementary Note 6 we consider landing. In order to land at the slowest possible speed, a still larger angle of attack than that corresponding to maximum endurance in engine-powered level flight and minimum sinking speed in gliding flight without engine power is optimal, namely the maximum practicable angle of attack, as near to a stall as is safe, which maximizes $L$ for a given airspeed $v$ at the expense of more-than-minimum sinking speed and even-lesser-than-maximum $L / D$. (The angle of attack should not be too near a stall, especially close to the ground, because there is not sufficient altitude to recover if an aircraft stalls too close to the ground.) See Ref. [2], pp. 178-180 and 208-209; Ref. [9], pp. 46-48, 54, and 156-157; Ref. [10], pp. 139-141, and Sects. X.1-X.2, XIV.4, XV.2, and XVI.4; Ref. [11], pp. 649-651; Ref. [12], pp. 26-27; Ref. [13], Chap. XVI; and Ref. [14], pp. 52, 54-55, and 72-75. It may be helpful to refer back to this Supplementary Note 6 when Supplementary Notes 9 and 10 concerning induced drag and skin-friction drag, respectively, especially the former, are encountered.

Supplementary Note 7: In Ref. [16] the concept of gravitational scale height (without the actual term being used) is stated specifically with respect to Earth's radius $R$, whereas in this present paper it is stated with respect to general $r \geq R$ (and also considering a borehole with respect to $r=0$ in a spherical gravitator of uniform density). The term "gravitational scale height" is analogous to "atmospheric scale height", the height of the top of 
an atmosphere if atmospheric density remained constant and did not decrease with increasing altitude. Atmospheric scale height is discussed, for example, in Sects. 2.2-2.3 of Ref. [16] and in Refs. [17] and [18]. There are also other scale heights, for example the scale height for boiling as discussed on pp. 203-204 of Ref. [16].

Supplementary Note 8: Drag and lift are often most fundamentally expressed as functions of Reynolds number and Mach number. See, for example, Ref. [2], Chap. 3, especially pp. 69-70, 89-99, and 108; also, in Chap. 5, pp. 165-181, and in Chap. 6, pp. 203-209. Especially relevant discussions concerning Mach number are provided on pp. 69-70 and 108, concerning Reynolds number on pp. 89-99 and 171, and concerning drag on pp. 172-176. Excellent explanations of Reynolds number are also provided on pp. 73-87 of Ref. [9] and in Sects. IV2-IV3 of Ref. [10], and of Mach number on pp. 77-78 and 106-107 of Ref. [9] and pp. 277-278 of Ref. [10]. General discussions concerning lift and drag are provided in Ref. [3], Chap. Thirteen; Ref. [9], especially Chaps. II-IV; Ref. [10], Chaps. V-VII; Ref. [11], Sects. 7.9-7.11, 12.11, and 14.10; and Ref. [13], Chaps. III-V. There are various classifications of drag, some classifications including, for example, interference drag, parasite drag, and shock-wave drag. (See the following articles: "Drag" (most recently revised in 2017) and "AP4ATCO - Drag - Types and Effects" (most recently revised in 2015) at https://www.skybrary.aero/index.php/Main_Page, and other SKYbrary articles concerning drag that are cited therein; also, "Drag (physics)", "Drag equation", and "Drag coefficient" (all most recently revised in 2019) at https://www.wikipedia.org, and other Wikipedia articles concerning drag that are cited therein. For simplicity, we construe these to be subsumed within pressure drag. A similar approach is taken, for example, on pp. 108-109 of Ref. [10] concerning interference drag, where interference is mentioned, but not classified as a separate type of drag. [Shock-wave drag (discussed in Chap. IV of Ref. [9], on p. ix of Ref. [10], and on pp. 58-60 and 182-183 of Ref. [14]), due to shock waves at and in the vicinity of Mach 1, does not occur for aircraft that do not reach speeds approaching Mach 1.]

Supplementary Note 9: Induced drag is a penalty that must be paid for lift. By Newton's third law of motion, a wing's action must be to deflect air downward in order to obtain the upward reaction of lift. The horizontal component of airflow encountered by an aircraft by virtue of its level (horizontal) aerodynamic flight is added vectorially to this downward component of airflow. Hence the direction of the lift force is not exactly vertical but instead is aligned at a (typically small) angle backward. This (typically small) backward component is the induced drag. According to the Prandtl-Munk formula, the minimum induced drag that a wing of span $b$ subject to airflow at speed $v$ must pay to generate lift $L$ is $D_{\text {induced,min }}=2 L^{2} / \pi \rho v^{2} b^{2}$. See Chaps. II-III (especially pp. 61-67) in Ref. [9], Chap. VI in Ref. [10], and the Wikipedia article "Lift-to-drag ratio" (most recently revised in 2019). Thus applying Equations (9) and (10): 


$$
\begin{aligned}
D_{\text {induced,min }} & =\frac{2 L^{2}}{\pi \rho v^{2} b^{2}}=\frac{2\left(\frac{1}{2} C_{L} A_{\text {wing,geom }} \rho v^{2}\right)^{2}}{\pi \rho v^{2} b^{2}}=\frac{C_{L}^{2} A_{\text {wing,geom }}^{2}\left(\rho v^{2}\right)^{2}}{2 \pi \rho v^{2} b^{2}} \\
& =\frac{C_{L}^{2} A_{\text {wing,geom }}^{2} \rho v^{2}}{2 \pi b^{2}}=\frac{C_{L}^{2} A_{\text {wing,geom }} \rho v^{2}}{2 \pi} \times \frac{A_{\text {wing,geom }}}{b^{2}} \\
& =\frac{C_{L}^{2} A_{\text {wing,geom }} \rho v^{2}}{2 \pi} \times \frac{b c}{b^{2}}=\frac{C_{L}^{2} A_{\text {wing,geom }} \rho v^{2}}{2 \pi} \times \frac{c}{b} \\
& =\frac{C_{L}^{2} A_{\text {wing,geom }} \rho v^{2}}{2 \pi \mathbf{A R}}=\frac{C_{L} A_{\text {wing, eff }} \rho v^{2}}{2 \pi \mathbf{A R}}=\frac{L C_{L}}{\pi \mathbf{A R}}=\frac{m g C_{L}}{\pi \mathbf{A R}},
\end{aligned}
$$

where $c$ is the average chord of an elliptically-shaped wing as is required for $D_{\text {induced }}=D_{\text {induced,min }}$ [see Ref. [2], pp. $72-74$ (especially p. 72); Ref. [9], pp. 65-67; and Ref. [10], Chap. IX (especially Sections IX.5-IX.7)], $A_{\text {wing,geom }}=b c$, the wing's aspect ratio is $\mathbf{A R} \equiv b / c$, and the last equality is justified because $L=m g$ in aerodynamic level flight. (Span, chord, and aspect ratio are defined at pp. 112-114 and 134-135 of Ref. [10] and pp. 55-58 of Ref. [13].) Typically at airspeeds $V$ at or near that corresponding to maximization of $L / D, C_{L}$ is approximately independent of air density $\rho$ and of $v$. But for an airplane or bird, especially one with short wingspan $b$ and small aspect ratio $\mathbf{A R}$, trying to land at the slowest possible speed $v$ well below that corresponding to maximization of $L / D$ and hence trying to maximize $L$ in the face of this slow $V$ whatever the cost in total drag $D$, induced drag $D_{\text {induced }}$ is likely the largest component of the total drag $D$. In such cases, owing to large $D_{\text {induced }}$, total drag $D$ is likely considerably larger than would typically be expected for the given $\rho v^{2}$. Recall Supplementary Note 6 and references mentioned in Supplementary Note 6. A simple qualitative explanation of induced drag, as well as its importance for birds with short wingspans especially if trying to land at the slowest possible speed, is provided on pp. 172-174 of Ref. [2]. For additional discussions concerning induced drag and related topics see additional relevant material in Refs. [2]-[15] and Supplementary Notes 1-6, and in references cited in Supplementary Notes 1-6.

In regards to reducing induced drag, we should mention wingtip devices. [See Refs. [21] [22] and the following articles: "Wing tip" and "Wingtip device" (both most recently revised in 2019) at https://www.wikipedia.org, and "Wing Tip Drag Reduction Devices" (most recently revised in 2017) at https://www.skybrary.aero/index.php/Main_Page. The idea has been around since the early 20th century (see especially Section 1 of Ref. [21]), but wingtip devices as generally construed came into actual use only in the 1970s, when improved wingtip-device design diminished offsetting factors sufficiently that the reduction of induced drag became a net positive factor, i.e., yielding a net improvement in airplane efficiency, both aerodynamically and financially (see especially Section 2 of Ref. [21]). Yet tapering of wings towards the tips can be construed as a simple wingtip device, because, for any given aspect ratio, this reduces the size of the wingtips to less than would have been the case 
with rectangular wings. And tapered wings were in actual use considerably earlier than the 1970s (see for example Sects. VI.4 and IX.7 of Ref. [10], p. 61 of Ref. [14], and pp. 63-64 of Ref. [15]). [By contrast, the aerodynamic fence, also known as the wing fence, blocks airflow towards the wingtips and serves a different purpose; hence it is not a wingtip device. See pp. 61-62 of Ref.[14], and the following articles: "Which is more effective a wing fence or winglet?" (date of most recent answer is 2018) at https://www.quora.com/Which-is-more-effective-a-wing-fence-or-winglet; Daskilewicz, M. (no date available, but date of most recent cited reference is 1989) "Stall Fences" (Georgia Tech Fixed Wing Design Class Wiki) at https://gtae6343.fandom.com/wiki/Stall Fences; and "Wing fence" (most recently revised in 2019) at https://www.wikipedia.org. (Note: the aforementioned quora website is also easily accessible via a Google search for: Which is more effective a wing fence or winglet.)]

Supplementary Note 10: Skin-friction drag $D_{\text {skinfric }}$ results from shear in airflow adjacent to surfaces parallel to the airflow-surfaces of an aircraft parallel to its direction of motion. Air sticks to such surfaces owing to its viscosity, so immediately at such surfaces the air is at rest, or very nearly at rest, relative to the aircraft. Beyond a boundary layer of typically small thickness $\mathcal{T}_{\text {boundary }}$, airflow relative to the aircraft approaches the airspeed $v$ of the aircraft-thus the shear $v / \mathcal{T}_{\text {boundary }}$. In short, skin-friction drag results from the viscosity of air rubbing against surfaces parallel to the airflow. According to simplified arguments, letting $\mu$ be the coefficient of viscosity of air and $A_{\|}$be an aircraft's surface area parallel to the airflow, there obtains in the simplest cases, as one might expect intuitively, the Stokes'-law-regime ${ }^{24}$ equation

$D_{\text {skinfric }}=\mu\left(v / \mathcal{T}_{\text {boundary }}\right) A_{\|}=\mu A_{\|} v / \mathcal{T}_{\text {boundary }} \quad$ (see for example pp. 75-78 of Ref. [9]). [In general $\mathcal{T}_{\text {boundary }}$ is not constant (see Section IV.5 of Ref. [10]), but an average value can be taken.] As per Sections IV.1-IV.2 (especially pp. 77-79) and Section V.5 of Ref. [10] drag in general and skin-friction drag in particular can often be expressed in the form $D_{\text {skinfric }}=\mathbf{C A} \rho v^{2}(\rho v l / \mu)^{-a}=\mathbf{C} A_{\|} \rho v^{2}(\mathbf{R e})^{-a}$, where $\mathbf{C}$ and $\mathbf{a}$ are dimensionless constants ( $\mathbf{C}$ could be a dimensionless function), $I$ is a characteristic length, and $\mathbf{R e} \equiv \mu / \rho v l$ is the Reynolds number. [Pressure drag and induced drag are effected via bulk flow rather than via viscosity, thus for them $a=-a=0$. Hence their functional dependencies on $\mathbf{R e}$ are subsumed within $\mathbf{C}$. For pressure drag (recall the first, second, and fifth paragraphs Section 3.1.1) $\mathbf{C}=\mathbf{C}(\mathbf{R e})=\left[C_{D}(\mathbf{R e})\right] / 2$ and $A_{\|} \rightarrow A_{\text {frontal,geom }}$; for minimum induced drag (recall Supplementary Note 9)

$\mathbf{C}=\mathbf{C}(\mathbf{R e}, \mathbf{A R})=\left[C_{L}(\mathbf{R e}, \mathbf{A R})\right]^{2} / 2 \pi \mathbf{A R}$ and $A_{\|} \rightarrow A_{\text {wing,geom }}$. The intuitive ${ }^{24}$ The Stokes' law regime obtains at small Reynolds numbers, typically taken as $\operatorname{Re}<1$, i.e., at Reynolds numbers much smaller than those corresponding to all aircraft flight except possibly that of the smallest flying insects. See for example: (a) Ref. [2], pp. 89-97 and 195-213; (b) Ref. [3], pp. 29-30 and 52; and (c) "Stokes' law" at https://www.wikipedia.org (most recently revised in 2019). See also the eighth paragraph of Section 3.1.1, the beginning of the first paragraph of Section 3.1.2, and Supplementary Note 10 of this present paper. 
result $\quad D_{\text {skinfric }}=\mu A_{\|} v / \mathcal{T}_{\text {boundary }} \quad$ obtains if $\quad \mathbf{C}=1, \quad a=1 \Leftrightarrow-a=-1$, and $l=\mathcal{T}_{\text {boundary }}$. Indeed only if $a=1 \Leftrightarrow-a=-1$ is $D_{\text {skinfric }}$ no function of $\rho$. But neither $\mathbf{C}$ nor a need equal 1. As per Chap. IV and especially Section V.5 of Ref. [10], more accurate consideration of skin-friction drag, even for the simple case of a flat rectangular plate of span $b$ perpendicular to the airflow and chord $c$ in the direction of (parallel to) the airflow assuming a laminar boundary layer, yields a result, as per Equations (5) and (6) on p. 106 of Ref. [10], that almost certainly could not be anticipated intuitively:

$D_{\text {skinfric }}=1.33 b\left(\mu \rho c v^{3}\right)^{1 / 2}$. Recognizing that, since both the top and the bottom of the flat plate are exposed to skin friction, $A_{\|}=2 b c$, this result can be expressed as $D_{\text {skinfric }}=0.665 A_{\|} \rho v^{2}(\rho v c / \mu)^{-1 / 2}=0.665 A_{\|} \rho v^{2}(\mathbf{R e})^{-1 / 2}$. If the boundary layer is turbulent rather than laminar, then even this result for $D_{\text {skinfric }}$ must be modified as per Equation (7) on p. 106 of Ref. [10]:

$$
\begin{aligned}
D_{\text {skinfric }} & =0.036 A_{\|} \rho v^{2}(\mathbf{R e})^{-1 / 5}=0.036 A_{\|} \rho v^{2}\left(\frac{\rho v l}{\mu}\right)^{-1 / 5} \\
& =0.036 A_{\|} \rho v^{2}\left(\frac{\mu}{\rho v l}\right)^{1 / 5}=0.036 A_{\|} \rho^{4 / 5} v^{9 / 5}\left(\frac{\mu}{l}\right)^{1 / 5} .
\end{aligned}
$$

A more exact result given a turbulent boundary layer is provided as per Equation (8) on p. 106 of Ref. [10]: employing this equation $D_{\text {skinfric }}$ probably must be evaluated numerically rather than analytically. Other formulas for $D_{\text {skinfric }}$ are provided in the Wikipedia article "Skin friction drag" (most recently revised in 2019) at https://www.wikipedia.org. (Span, chord, and aspect ratio are defined on pp. 112-114 of Ref. [10] and on pp. 55-58 Ref. [13]. Boundary layer is discussed in some detail on pp. 55-58 of Ref. [9], and on pp. 87-96 and in Section IV.5 of Ref. [10].) Note that air density $\rho$ appears in the more accurate equations for $D_{\text {skinfric }}$ but not in the intuitive one $D_{\text {skinfric }}=\mu A_{\|} v / \mathcal{T}_{\text {boundary }}$, that intuitively $D_{\text {skinfric }}$ is directly proportional to $\mu$ but not so according to more accurate equations, and that only the magnitude of $A_{\|}$matters in the intuitive equation for $D_{\text {skinfric }}$ while both the magnitude and the shape of $A_{\|}$matter in more accurate ones. Also note that neither the intuitive result for $D_{\text {skinfric }}$ nor the more accurate ones are even approximately proportional to $\rho v^{2}$. The intuitive $D_{\text {skinfric }}=\mu A_{\|} v / \mathcal{T}_{\text {boundary }}$ increases with increasing $v$ only linearly rather than as $v^{2}$. As per Equations (5) and (6) on p. 106 of Ref. [10], if the boundary layer is laminar $D_{\text {skinfic }}=1.33 b\left(\mu \rho c v^{3}\right)^{1 / 2}$ increases with increasing $v$ only as $v^{3 / 2}$ rather than as $v^{2}$. And as per Equation (7) on p. 106 of Ref. [10], if the boundary layer is turbulent $D_{\text {skinfric }}=0.036 A_{\mid} \rho v^{2}(\mathbf{R e})^{-1 / 5}=0.036 A_{\|} \rho^{4 / 5} v^{9 / 5}(\mu / l)^{1 / 5}$ increases with increasing $v$ only as $v^{9 / 5}$ rather than as $v^{2}$. By contrast, given the paramount $\rho v^{2}$ functional dependency of pressure drag, and also-recall Supplementary Note 9-of induced drag, they are disposed to increase with increasing $V$ as $V^{2}$. Hence skin-friction drag becomes less important relative to pressure drag and to induced drag with increasing $V$, or more fundamentally with increasing Reynolds number Re. The coefficient of skin-friction drag is 
typically small, a few times $10^{-3}$. Hence skin-friction drag is often only a small fraction of the total drag $D$ as given by Equation (7) of this present paper. See pp. 87-97 (especially pp. 93-94) of Ref. [9], and most especially Sects. V.5 and VI.2 of Ref. [10]. Often the functional dependence of skin-friction drag is not stated explicitly, and skin-friction drag is instead merely incorporated into Equations (7) and (8) of this present paper via a contribution to $C_{D}$ that is often small, and even if not small can still be thus incorporated within $C_{D}$, in accordance with the second and eighth paragraphs of Section 3.1.1 of this present paper. For underwater airplanes as discussed in Section 3.3.2 of this present paper, substitute "water" for "air"; most generally, substitute "fluid” for "air". See also pp. 172-174 of Ref. [2], Refs. [3]-[8], and Sects. 7.10, 12.11, and 14.10 of Ref. [11].

Supplementary Note 11: The transition from laminar to turbulent flow is discussed on pp. 78-79 of Ref. [9], in Section V.3 of Ref. [10], and in Figs. 7.7 (a) and 7.7 (b) on pp. 400-401 and Sects. 14.10.5-14.10.6 of Ref. [11]. A concise survey of golf-ball aerodynamics is provided in Ref. [23]. For more detailed discussions see Refs. [24] [25], which are cited in Ref. [23]. See also the excellent, easily-understandable discussion of golf-ball aerodynamics in Ref. [26]. Assists for laminar-to-turbulent transition as employed, for example, in fluid-dynamic modeling and in small aircraft are discussed on pp. 107-108 and 208-209 of Ref. [2].

Supplementary Note 12: A sharp rise in $C_{D}$ as Mach 1 is approached from below followed by a sharp dip in $C_{D}$ at values of $V$ slightly above Mach 1 obtains for airplane wings. See pp. 128-129 of Ref. [9]. But for unstreamlined bodies only a sharp rise in $C_{D}$ as Mach 1 is approached from below may obtain, with the dip in $C_{D}$ at values of $v$ slightly above Mach 1 being much less pronounced than for (streamlined) airplane wings, and perhaps in some cases even nonexistent. See Section 15.10 of Ref. [11]. [Shock-wave drag (discussed in Chap. IV of Ref. [9], on p. ix of Ref. [10], and on pp. 58-60 and 182-183 of Ref. [14]), is due to shock waves at and in the vicinity of Mach 1 . See also the articles "Wave drag" (most recently revised in 2019) at https://www.wikipedia.org and "Wave Drag" (most recently revised in 2017) at https://www.skybrary.aero/index.php/Main Page.]

Supplementary Note 13: Discussions and data pertinent to the U. S. Standard Atmosphere are provided in Chap. II (especially p. 19) of Ref. [13]; Sects. 2.2, 2.3, and 2.55 of Ref. [16]; Section 1.3.4 of Ref. [17]; and pp. 18-28, 424-428, and 487 of Ref. [27]. Meticulously detailed data, including discussions, tables, and charts, concerning atmospheric density and other atmospheric properties as a function of altitude are provided in Refs. [29] [30] [31]. Somewhat less recent data, but also meticulously detailed, including discussions, tables, and charts, concerning atmospheric density and other atmospheric properties as a function of altitude are provided in Ref. [32]. Somewhat abbreviated but still more than adequate discussions, tables, and charts concerning atmospheric density and 
other atmospheric properties as a function of altitude are provided in Refs. [33] [34].

Supplementary Note 14: The average surface atmospheric pressure at low altitudes on Mars is $\approx 1 / 120$ of that on Earth. But owing to both the higher average molecular weight of Mars' largely- $\mathrm{CO}_{2}$ atmosphere and its lower average temperature, the average surface atmospheric density at low altitudes on Mars is $\approx$ 1/70 of that on Earth [35] [36] [37] (see also Supplementary Note 14). See relevant data provided in Refs. [35] [36] [37] and in the following articles (both most recently revised in 2019) at https://www.wikipedia.org: "Earth" and "Mars". Reviews of Mars aircraft are provided in the following Wikipedia articles (both most recently revised in 2019) at https://www.wikipedia.org: "Mars aircraft" and "JPL Mars Helicopter Scout".

Supplementary Note 15: The hand-thrown distance records for discuses, Frisbees, Aerobies, and boomerangs are provided in the following articles (all most recently revised in 2019) at https://www.wikipedia.org: "Discus throw", "Frisbee", "Aerobie", and "Boomerang". Concerning the hand-thrown distance record for Frisbees, see especially http://www.wfdf.org/worldrecords (left-click on "Outdoor Distance" under "Distance"). The hand-thrown distance records for baseballs and golf balls are provided in the following articles at https://www.wikipedia.org ("Glen Gorbous" and "Roald Bradstock", respectively, both most recently revised in 2019. The hand-thrown distance record for javelins is provided in the following articles (both most recently revised in 2019) at https://www.wikipedia.org: "Uwe Hohn" and "Javelin throw". Sports-ball aerodynamics is discussed on pp. 31-34 and 40 of Ref. [9], in Refs. [23] [24] [25] [26] [39] [40], in Supplementary Note 11 and in the references cited therein, and in the Wikipedia article "Golf ball" (most recently revised in 2019) at https://www.wikipedia.org. Javelin aerodynamics is discussed in Ref. [41]. Discus aerodynamics is discussed in Refs. [42] [43]. Wikipedia articles concerning world-record-setting discus-throw athletes (all at https://www.wikipedia.org, all most recently revised in 2019) include "Jürgen Schult", "Gabriele Reinsch", and "Martina Hellmann".

Supplementary Note 16: For symmetrical hand-thrown aircraft such as Frisbees, Aerobies, or discusses, it is the magnitude $\left|v_{\text {air }}\right|$ of $v_{\text {air }}$ that alone determines whether or not aerodynamic level flight can be sustained, irrespective of the sign of $v_{\text {air }}$. (The symmetry must be at least bilateral with respect to the forward/backward direction.) If $0 \leq v_{\text {ground }}=v_{\text {air,min }}<v_{\text {wind }}$, $v_{\text {air }}=v_{\text {ground }}-v_{\text {wind }}=v_{\text {air,min }}-v_{\text {wind }}<0$, and

$\left|v_{\text {air }}\right|=v_{\text {wind }}-v_{\text {ground }}=v_{\text {wind }}-v_{\text {air,min }}>0$. Aerodynamic level flight of a symmetrical hand-thrown aircraft such as a Frisbee, Aerobie, or discuss can then be maintained if $\left|v_{\text {air }}\right| \geq v_{\text {air,min }}$, which can obtain if $v_{\text {wind }} \geq 2 v_{\text {air,min }}$. The analyses for unsymmetrical hand-thrown aircraft such as hand-thrown (e.g., paper or balsa) gliders if $0 \leq v_{\text {ground }}=v_{\text {air,min }}<v_{\text {wind }} \Leftrightarrow v_{\text {air }}<0$ is more complex, and we do not attempt it. $v_{\text {air }}<0$ corresponds to an aircraft flying backwards. For a symmetrical aircraft (hand-thrown or otherwise) forward and backward flight are 
aerodynamically equivalent-but of course not so for unsymmetrical aircraft (hand-thrown or otherwise).

Supplementary Note 17: See the following articles (all most recently revised in 2019) at https://www.wikipedia.org: "Friction", "Rolling resistance", "Energy efficiency in transport", "Magnetic levitation", "Maglev", and "Inductrack". (While traditional railroad transportation is more energy-efficient at low to moderate speeds, maglev trains almost completely abolish wear on the tracks, owing to their lack of mechanical contact with the tracks: see the last three Wikipedia articles cited in this Supplementary Note 17.) See also the following articles at The Engineering Toolbox website https://www.engineeringtoolbox.com: "Friction and Friction Coefficients" (most recently revised in 2004) at https://www.engineeringtoolbox.com/friction-coefficients-d_778.html. and "Rolling Resistance" (most recently revised in 2008) at https://www.engineeringtoolbox.com/rolling-friction-resistance-d_1303.html.

Supplementary Note 18: Ground effect is discussed in the following articles: "Ground Effect" (most recently revised in 2017) at the SKYbrary website https://www.skybrary.aero/index.php/Main_Page; also, "Ground effect (aerodynamics)" and "Ground-effect vehicle" (both most recently revised in 2019) at https://www.wikipedia.org. Intuitively, one might attribute the ground effect for an aircraft near the ground as being due to compression of air near the ground, thus creating a lift-augmenting cushion of air under the aircraft that helps to support the aircraft. The wings, and possibly the aircraft as a whole, being inclined slightly upwards towards the direction of flight helps in this compression of air near the ground and thus in the creation of this air cushion. (The ground is usually a land surface, but could be a smooth water surface.) But this is only partially correct. Ground effect is a more complex, multifaceted, phenomenon. A perhaps less intuitively obvious contribution to the ground effect for aircraft arises because the proximity of the ground does not allow vortices at the wing tips to fully form, thus reducing induced drag. Thus ground effect results not only in increased lift but also in decreased drag. A given lift is produced at a smaller angle of attack with ground effect than without it. This is consistent with induced drag being smaller with ground effect than without it, because it corresponds to lift being directed at a smaller angle backwards (recall Supplementary Note 9). Thus at any given angle of attack ground effect results not only in increased lift but also in decreased drag. Hence a higher $L / D$ ratio obtains at any given angle of attack with ground effect than without it owing to both increased lift and decreased drag. Also, the stalling angle of attack (recall Supplementary Note 6) is larger with ground effect than without it. 